\title{
Über Darminvaginationen.
}

Kritik der geltenden Lehre von der Invaginatio ,ileocoecalis" und der üblichen Nomenklatur; nebst kasuistischen Beiträgen.

Von Privatdozent Dr. Hermann Matti, Chirurg am Jenner-Kinderspital in Bern. (Dir. Prof. Dr. StooB.)

(Mit 6 Abbildungen.)

Unter den verschiedenen Formen des Darmverschlusses im frühen Kindesalter spielt die Intussuszeption nach übereinstimmenden Feststellungen aller Autoren die bedeutendste Rolle; zudem entfallen nach der großen Statistik von W e i $\beta 55$ Proz. aller Invaginationsfälle auf Neugeborene und Kinder im Säuglingsalter, weitere 26 Proz. auf Kinder vom zweiten Jahre bis zur Pubertät. Diesen Zahlen gegenüber treten die I9 Proz. Darmeinschiebungen bei Erwachsenen ganz bedeutend in den Hintergrund. Diese Feststellungen haben ihr ganz bestimmtes Interesse. Einmal zeigen sie uns, da $\beta$ bei Kindern offenbar bestimmte Dispositionen für diesen pathologischen Vorgang vorhanden sein müssen, deren Studium und möglichste Abklärung in erster Linie den Weg zur Erklärung und zum Verständnis dieser merkwürdigen pathologischen Konsequenz der Darmperistaltik zu bahnen geeignet erscheint. Neben diesem allgemeinen Interesse der Frage für die Darmpathologie liegt sodann die große Bedeutung der Invaginationen in der überaus ernsten Prognose des Darmverschlusses und ganz besonders dieser Form bei Kindern, eine Tatsache, die den Pädiater und den Chirurgen in gleichem Maße beschäftigt. Man wird diese Darstellung mit Rücksicht auf die bekannten Mitteilungen Hirchsprungs über seine Resultate bei 107 Fällen von Invagination bei Kindern vielleicht als zu pessimistisch gefärbt halten. Bekanntlich behauptet $\mathrm{H}$ ir s c hsprung, unter seinen Fällen 60 Proz. durch unblutige Me- 
thoden geheilt zu haben, wobei namentlich darauf hingewiesen wird, da $\beta$ von 44 Kindern, bei denen die Krankheit weniger als 24 Stunden gedauert hatte, 70 Proz. geheilt wurden, während bei längerer Krankheitsdauer die Heilungsziffer nur noch 30 Proz. betrug. Ganz abgesehen davon, daß $\mathrm{H}$ i r s ch s p r u $\mathrm{ng}$ in der glücklichen, für die meisten Kinderärzte leider nicht realisierbaren und deshalb auch nicht maßgebenden Lage war, einen ungewöhnlich hohen Prozentsatz seiner Patienten in einem günstigen Frühstadium zu behandeln, stimmen seine Angaben, worauf namentlich $\mathrm{W}$ il m s aufmerksam macht, mit den allgemeinen Erfahrungen nicht überein. So verzeichnen die großen Statistiken bei unblutiger Behandlung nur durchschnittlich 30 Proz. Heilungen. $\mathrm{Clu} \mathrm{b} \mathrm{be} \mathrm{konnte}$ von 49 Invaginationen nur 4, also 8 Proz., mittels Einläufen reponieren, während $\mathrm{R}$ ig by unter 53 Fällen des Londoner Hospitals keine einzige unblutige Reduktion zu verzeichnen hat, vielmehr alle Patienten der Laparotomie unterwerfen mußte. $\mathrm{Clu} \mathrm{b} \mathrm{be} \mathrm{macht} \mathrm{im} \mathrm{ferneren} \mathrm{darauf} \mathrm{auf-}$ merksam, daß man sich bei den unblutigen Repositionen durch zeitweise Nachuntersuchungen davon überzeugen müsse, ob kein Rezidiv eingetreten sei. Noch auffälliger kontrastieren die Angaben von Kirmisson mit den Resultaten Hirschsprungs. $\mathrm{K}$ i $\mathrm{rm}$ is s o $\mathrm{n}$ gibt an, daß von den Invaginationsfällen, die er im Laufe von 18 Jahren beobachtete, sämtliche bis auf einen starben; dieser einzige glücklich verlaufene Fall betraf ein Kind, das sechs Stunden nach Beginn der Erkrankung in Behandlung kam. Dieser auffällige Gegensatz hinsichtlich der Möglichkeit unblutiger Behandlung der Darmeinscheidung bei Kindern und damit auch der Gegensatz in der Prognose, wie er sich aus den Berichten der meisten Autoren gegenüber den Mitteilungen $\mathrm{H}$ irschsprungs ergibt, muß gewisse $Z$ weifel hervorrufen, ob tatsächlich alle von $\mathrm{Hirschsprung}$ geheilten Fälle von Darminvagination wirkliche Einscheidungen waren. Wilms schließt sich diesem von $\mathrm{Bra}$ u $\mathrm{n}$ ausgesprochenen $Z$ weifel an und macht uns aufmerksam, daß nach $\mathrm{H}$ i r s ch s p r ung habituelle Obstipation bei Kindern ein wichtiges ätiologisches Moment für die Intussuszeption darstelle, während diarrhoische Zustände nur geringe Bedeutung hätten, was den allgemein gültigen Anschauungen widerspreche; aus diesem Grunde liege die Annahme 
nahe, daß in manchen Fällen die Erscheinungen der Invagination auf chronischer Obstipation beruhten, um so mehr, als wir über die Frequenz der wichtigsten pathognomischen Symptome, den Nachweis eines Tumors, sowie den Abgang von blutigem Schleim gar keine Angaben erhalten. Sehr merkwürdig ist auch der Umstand, da $\beta$ nach $\mathrm{Hirschsprung}$ acht Neuntel der Invaginationen Ileocöcal- und reine Dickdarminvagination betrafen, während nur der neunte Teil Dünndarmeinscheidungen waren; nach den an anderem großen Material gewonnenen Erfahrungen ist das Verhältnis der Dünndarmeinscheidungen zu den Dickdarminvaginationen 25 Proz. zu 75 Proz. Auch dieses auffällige Überwiegen der Dickdarminvaginationen (inklusive ileocöcale) muß unsere Zweifel in ganz bestimmter Hinsicht erregen. Man kann diesen Ausführungen von $\mathrm{W}$ i $1 \mathrm{~m} \mathrm{~s}$, die unter den neueren Autoren auch von $\mathrm{K} l$ e $\mathrm{m} m$ und $\mathrm{H}$ a $\mathrm{n}$ s e $\mathrm{n}$ geteilt werden, nur zustimmen und darf mit aller Berechtigung sagen, daß die Darminvagination bei Kindern nicht das prognostisch erfreuliche Bild darbietet, wie es uns aus der Hirschsprungschen Statistik entgegenblickt, sondern daß jede, auch chronische Intussuszeption für ihren Träger eine große, direkte Lebensgefahr in sich birgt und da $B$ die Prognose entsprechend ernst zu stellen ist, was, abgesehen vom Gesagten, daraus hervorgeht, da $B$ nur in etwa 33 Proz. der Fälle Heilung durch unblutige Maßnahmen zu erzielen ist, und daß nach $\mathrm{C}$ h e n e y etwa 60 Proz. der Kinder im ersten Lebensjahre ihrer Invagination erliegen. Es hat meines Erachtens ein großes praktisches Interesse, auf diese Tatsachen hinzuweisen, namentlich aus dem ferneren Grunde, weil im Allgemeinen die Kinder mit Invaginationen erst in Behandlung kommen, wenn schon mehr als 24 Stunden verflossen sind seit dem Beginne der Erscheinungen, und nach diesem Zeitpunkte hatte ja auch $\mathrm{Hirschsprung}$ nur eine Heilungsziffer von 30 Proz. Da es sich bei Kindern beinahe ausnahmslos um akute Invaginationen handelt - die chronischen machen nach $W$ e i $B$ nur 3 Proz. aus - sind überhaupt die Chancen, daß unblutige Maßnahmen zum Ziele führen, ganz verschwindende. Verliert man in solchen Fällen noch kostbare Zeit mit allen möglichen Repositionsversuchen, so verpaßt man damit die beste Zeit für eine Frühoperation, als welche wir die operative Desinvagination zu betrachten haben, die im Allge- 
meinen nur bei Fällen gelingt, bei denen die Einscheidung nicht länger als 24 . Stunden besteht. Ist die Einklemmung eine sehr intensive, so kann schon in den ersten Stunden eine Desinvagination ohne Darmläsion unmöglich sein, während allerdings bei chronischen Intussuszeptionen noch nach Tagen und Monaten eine Reduktion ausführbar sein kann. Daß man in frischen Fällen eine Taxis der Invaginationen in Narkose, eventuell unter $\mathrm{Zu}$ hilfenahme von Wassereinläufen, versuchen wird, ist selbstverständlich; nur wird man mit nutzlosen Versuchen die Fälle nicht verschleppen. Denn wenn sich auch die Resultate der operativen Behandlung im Allgemeinen im Laufe der Jahre außerordentlich gebessert haben, so zeigen doch auch die neuesten Statistiken, daß die operative Heilungsziffer vom zweiten Tage nach der Einscheidung an rapid sinkt. Das beruht auf der bedeutend schlechteren Prognose der Resektion gegenüber der operativen Desinvagination, die ja nur in Frühfällen Erfolg verspricht. So beträgt nach $\mathrm{Gib}$ s on die Heilungsziffer am ersten und zweiten Tage nach der Einscheidung 59 Proz., am dritten Tage 38 Proz., vom dritten bis sechsten Tage 28 Proz., nach dem sechsten Tage o Proz. Bei Kindern unter drei Monaten war die Mortalität 82 Proz., bei Kindern unter einem Jahre 65 Proz. Wenn wir die Resultate der operativen Desinvagination und der Resektion nach der letzten großen Statistik von $W$ e i $B$ vergleichen, so sehen wir, da $B$ beim Neugeborenen und Säugling die Desinvagination 58 Proz., die Resektion 47 Proz. Heilungen ergab, bei Patienten im Kindesalter und bis zur Pubertät die Desinvagination 70 Proz., die Resektion 57 Proz. Die Besserung der Resektionsresultate mit den Fortschritten der aseptischen Technik ist eine ganz hervorragende. Im Jahre $\mathrm{I} 885$ verzeichnete $\mathrm{B} \mathrm{r}$ a u $\mathrm{n}$ bei den Resektionen Ioo Proz. Mortalität, Rydygier I895 unter I2 Fällen 25 Proz., Gi bs o n r897 unter 45 Fällen 15,5 Proz. W e i $B$ berechnet in seiner schon erwähnten Statistik über 322 Fälle aus den Jahren r894 bis 1898 unter den Resektionen 57 Proz. Heilungen. W a l t e r $\mathrm{m}$ a $\mathrm{n} \mathrm{n}$ fand von $\mathrm{I} 898$ - I902 auf 22 Heilungen bei totaler oder partieller Resektion 7 Todesfälle, was einer Mortalität von 32 Proz. gleichkommt. Noch besser sind die Resultate v. E is el s b e r g s , der bei I 2 Totalresektionen 9 Heilungen erzielte, was einer Mortalität von nur 25 Proz. entspricht. So erfreulich diese unleugbare 
Besserung der Resektionsresultate auch ist, so kann man sich des Eindrucks nicht erwehren, daß diese angeführten Zahlen den tatsächlichen Verhältnissen nicht entsprechen, aus dem ganz naheliegenden Grunde, weil aus einzelnen oder mehreren ungünstig verlaufenen Resektionen ein Impuls zu publizistischer Tätigkeit gewöhnlich nicht resultiert. Fs steht ganz a u Ber allem $Z$ weifel, da $B$ die Mortalitätsziffern für die Darmresektion bei Invaginationen, wie sie a d den großen Statistiken hervorgehen, viel zu niedrig gegriffen sind. Daß einzelne tüchtige Chirurgen bessere Resultate haben, kann an diesem Faktum nichts Wesentliches ändern. Denn das Schicksal der zur Resektion gelangenden Fälle entscheidet sich oft ganz außerhalb der Sphäre operativer Technik, d. h. es ist oft schon vor der Resektion entschieden und dem Chirurgen fällt nur noch die undankbare Aufgabe $z u$, mit der ultima ratio einer Resektion das Schicksal der kleinen Patienten zu beschleunigen. Es ist ja zur Genüge bekannt, wie empfindlich Kinder gegenüber größeren abdominellen Eingriffen sind, namentlich, wenn infolge des schon längere Zeit bestehenden Darmverschlusses der Kollaps vor der Türe steht.

So bietet die Invagination bei Kindern trotz der Hirschsprungschen Ausnahmestatistik im allgemeinen doch noch ein trübes Bild dar, und die Fälle von Intussuszeption sind schon aus diesem Grunde von größtem klinischen Interesse. Eine Bereicherung der Kasuistik kann nur dazu beitragen, die tatsächlichen Verhältnisse abklären zu helfen und wir halten uns deshalb für berechtigt, einen Fall von Invagination bei einem Kinde, den wir im Laufe des letzten Jahres am Jenner-Kinderspital zu operieren Gelegenheit hatten, und der in diagnostischer und therapeutischer Hinsicht einiges Interesse bietet, an dieser Stelle mitzuteilen. Bei dieser Gelegenheit sind wir in der Lage, noch über vier weitere Fälle zu berichten, die von unserem Vorgänger am Jennerspital, Prof. T a v e 1, operiert wurden und die er uns gütigst überlassen hat. Wir bringen zunächst die gekürzten Krankengeschichten, deren klinische Diskussion anschließend folgt. Verschiedene Kontroversen werden uns Anlaß geben, einzelne Punkte der Diagnostik, Pathogenese und Therapie, sowie namentlich auch 
die Frage einer rationellen Klassifikation der Invaginationen, die unseres Erachtens noch zu Mißverständnissen anlaßgebende Unklarheiten darbietet, eingehender $\mathrm{zu}$ besprechen.

I. Rudolf W. $9 \mathrm{M}$ on at $\mathrm{e}^{2}$. Aufnahme 28. II. I9Io.

A n a m nese: Das Kind hat seit zwei Tagen keine Stuhlentleerungen mehr gehabt; am Tage vor der Spitalaufnahme akute Erkrankung mit heftigen Bauchschmerzen - Schreien, Krämpfe, leichte Auftreibung des Abdomens - dann erfolgten einige blutige Stühle, später blutig-schleimige Entleerungen. Heute morgen, also etwa dreißig Stunden nach Beginn der Erkrankung, begann das Kind zu erbrechen. Seither hat sich das Erbrechen mehrmals wiederholt.

S t a t u s: Patient sieht verhältnismäßig gut aus, ist aber deutlich apathisch, jammert zeitweise und zeigt Zeichen intensiver Schmerzen, wobei es die Beinchen anzieht.

Abdomen nur wenig aufgetrieben; nach links und unterhalb vom Nabel sieht man einige geblähte Darmschlingen sich abzeichnen, ohne sichtliche Darmsteifung. Die Bauchdecken sind nicht gespannt! D i e Ileocöcalgegend scheint etwas leerer als die sy m metrisch gelegene Partie $1 \mathrm{inks}$. Perkussion ergibt keine auffälligen Verhältnisse, weder Dämpfung noch Tympanie oder Metallklang. Kein Hochstand der Leber. D i e P a l p a t i o $\mathrm{l} l \ddot{\mathrm{a}} \beta \mathrm{t}$ weder Resistenz noch Tumorbildung nachweisen; auch scheint keine Druckempfindlichkeit zu bestehen. Auf einen Wassereinlauf in das Rektum entleert sich mit der Flüssigkeit etwas blutig tingierter Schleim, jedoch keinerlei Stuhlpartikel. Dagegen gehen Winde ab! Der per rectum untersuchende Finger stößt am Utbergang zur Flexura sigmoidea auf einen scharfen Kontraktionsring, der sich zeitweise löst. Irgendein Tumor im Darm nicht zu fühlen. Eine bimanuelle Exploration des Unterbauches und Beckens hat ebenfalls ein negatives Resultat. Am Finger haftet dicker, weißer Schleim.

Di a g n o se: Die Anamnese legt den Gedanken an eine Invagination nahe; aus dem objektiven Untersuchungsbefund läßt sich jedoch die Diagnose Intussusception nicht genügend stützen. Es fehlt die Bauchdeckenspannung, es fehlt der Nachweis eines Invaginationstumors. Gleichwohl wird mit Rücksicht auf die Anamnese: plötzlicher Beginn mit Schmerzen, blutige und blutig-schleimige Darmentleerungen, Erbrechen - sowie unter Verwertung der vorhandenen objektiven Symptome: Auftreibung mit Darmzeichnung, schleimige Abgänge aus dem Rektum mit leicht blutiger Tinktion, ohne Beimischung von KotPartikeln, Abflachung der Ileocöcalgegend (Dancesches Symptom) die

1) Fälle Nr. I, 3, 4 und 5 stammen aus dem Kinderspital und wurden von Direktor, Prof. Dr. Stoo $\beta$, dem Chirurgen zugewiesen. 
Diagnose auf eine Darminvagination gestellt. Das Fehlen eines Tumors wird durch die Annahme erklärt, daß das Cöcum nach oben ins Colon invaginiert und so unter dem Rippenbogen versteckt sei. Auch könnte eine reine Dünndarminvagination vorliegen. Aus prognostischen Erwägungen ist die sofortige Operation unbedingt indiziert.

Operation: Äthernarkose; vor der Laparotomie wird in guter Narkose nochmals palpiert. Irgendein Tumor oder eine Resistenz im Abdomen ist nicht nachweisbar. Die Ileocöcalgegend erscheint abnorm leer.

Mediane Incision vom Nabel zur Symphyse; aus der Peritonealhöhle entleert sich klares Exsudat. Sofort quellen einige stark geblähte und venös-hyperämische Dünndarmschlingen vor. Zunächst findet sich eine Io $\mathrm{cm}$ lange Bride, welche von der Radix mesenterii nach dem Nabelring zieht; Excision dieses Stranges. Da dieser Befund zur Erklärung der Symptome nicht ausreicht, wird der Dünndarm eventriert, der sich etwa zur Hälfte als normal, zur Hälfte als gebläht erweist. Nun findet man rechts neben der Wirbelsäule, weit o b e $n$ un $t$ e $r$ d e m Rippenboge $n$, ungefähr im Niveau der rechten Niere, einen hühnereigroßen Tumor, der sich mühsam vorziehen läßt und als Invaginationstumor sich erweist. Die Geschwulst läst sich schließlich vor die Bauchwunde luxieren. Die Scheide des Tumors entspricht dem oberen Teile des Colon ascendens und der Flecura coli dextra. Durch leisen $\mathrm{Zug}$ am Invaginatum und sorgfältigen Druck auf das Intussuscipiens gelingt es zunächst, das Cöcum mit dem Appendix zu desinvaginieren; dann kann ein etwa $30 \mathrm{~cm}$ langes Stück Ileum ziemlich leicht desinvaginiert werden. Schließlich bleibt noch eine $10 \mathrm{~cm}$ lange, an der Umbiegungsstelle stark infiltrierte Dünndarmschlinge im Cöcum, die sich nur schwer, aber schließlich durch methodischen Zug und Druck doch ohne Verletzung entwickeln läßt. Das Desinvaginationshinclernis lag an der Stelle der primären Invagination von Ileum in Ileum, $3 \mathrm{~cm}$ oralwärts von der Ileocöcalklappe. Die betreffende Darmpartie ist auf eine Strecke von 5-6 cm intensiv hämorrhagisch infiltriert und ödematös, zeigt jedoch keine Verletzung der Serosa; Kontraktilität und Pulsation der Arterien erhalten. Im ebenfalls infiltrierten Mesenterium einige Ekchymosen. Der Darm erholt sich unter Irrigation mit warmer Kochsalzlösung und wird deshalb reponiert. Der Wurmfortsatz ist um I $80^{\circ} \mathrm{um}$ seine Längsachse torquiert und mit seinem Mesenteriolum breit an dem verlängerten Mesocöcum verwachsen; er wird in typischer Weise abgetragen. Getrennte Peritoneal-Fasciennaht, fortlaufende Hautnaht.

Verlauf: Die Temperatur steigt am I. III. auf $39,7^{\circ}$, a m 2. III. e rreich t sie $39,6^{\circ}$, um am dritten Tage nach der Operation auf $37,4^{\circ}$ abzufallen. 36 Stunden post op. gehen Stuhl und Winde $a b$; die erste Stuhlentleerung enthält noch Blut. Unter vorsichtiger Diät normaler Verlauf; das $\mathrm{K}$ ind wird a m I6. III. geheilt entlassen. 
2. Viktor H. 4 Mon a te. Eintritt 24. VI. I902.

Patient litt während einigen Wochen an Verdauungsstörungen. Seit 24 Stunden Erbrechen und Stuhlverhaltung.

$S t$ a tu s: In der linken Bauchseite, dem Colon descendens entsprechend, fühlt man eine längliche Geschwulst; Abdomen nur wenig aufgetrieben, keine Tympanie; starke Peristaltik. Per rectum fühlt man die unteren Partien der Geschwulst durch die Rektalwand hindurch.

$\mathrm{D}$ i a $\mathrm{g} \mathrm{n}$ o s e: Invagination.

$\mathrm{O}$ p e $\mathrm{r}$ a $\mathrm{t}$ i o $\mathrm{n}$ 25. VI. morgens: Schräge Incision links über dem Tumor; man gelangt sofort auf die Geschwulst, welche mit ihrem unteren Pol in der Fossa iliaca sinistra liegt und nach oben bis in Nabelhöhe reicht. Es handelt sich um eine Invagination des Ileums und Cöcums ins Colon ascendens und transversum. Die Reposition kann nicht ausgeführt werden; es zeigt sich, daß das Ileum an der Stelle des Eintritts in das Colon (Umschlagstelle) um seine Achse torquiert und entsprechend sekundär verändert ist. Deshalb Resektion und End-zuEnd-Anastomose zwischen dilatiertem Ileum und Colon. Bauchwandnaht.

Verlauf: Die Temperatur steigt a uf $39,2^{\circ}$, dann setzt ein Kollaps ein, in dem das Kind stirbt.

3. A $1 \mathrm{fred} \mathrm{B}$. $7 \mathrm{M}$ on at e. Eintritt 8. VI. I907.

Das Kind wurde bis zum 5.VI. von der Mutter gestillt; a n d i e s e m Tage zum erstenmal Ernährung mit Kuhmilch und aufgeweichtem Brot. Am Abend Erbrechen gelber, schleimiger Massen; Diarrhöe. Vom Arzte verordnetes Kalomel war ohne Effekt; dagegen erfolgte am 7. VI. auf Klysma ein brauner, übelriechender Stuhl. Am folgenden Tage blieben Klysmen ohne Wirkung. Magenspülung.

$\mathrm{S} t$ a $\mathrm{t}$ u s: Patient sieht verfallen aus, hat einen ängstlichen Gesichtsausdruck und wimmert bei der Untersuchung. Leib stark aufgetrieben; Darmzeichnung. Hochstand der Lebergrenze; auch das Herz nach oben verdrängt. Die Spannung der Bauchdecken ist so hochgradig, daß die Palpation negativ ausfällt, während der zuweisende Arzt einen Tumor gefühlt hatte. Rektum leer; hoch oben nach links zu eine deutliche, aber nicht abgrenzbare Resistenz.

O peration: Medianschnitt vom Nabel zur Symphyse; Dünndarmschlingen dilatiert und hyperämisch. Man findet median und rechts einen Tumor, der sich als eine Invaginatio ,,ileo-coecalis" erweist. Es gelingt, $z u$ desinvaginieren. Bauchwandnaht.

Verlauf: Die Temperatursteigt a m 9.VI. a u f $39^{\circ}$, erhebt sich nach vorübergehender Senkung am I2. VI. nochmals auf $38,3^{\circ}$, um dann zur Norm zurückzukehren. Der Darm ist schon am Tage nach der Operation wieder durchgängig und das Kind erholt sich unter vorsichtiger Diät sehr rasch. 25. VI. En tla s s ung. Vol1ständig geheilt. 
4. Fried a M. 21/2 J a hre. Eintritt I2. VI. Igog.

Am 5. VI. klagte das Kind über Schmerzen im Bauch; am folgenden Tage Erbrechen und Fortdauern der Schmerzen, Stuhlverhaltung. Ein am 8. VI. konsultierter Arzt diagnostiziert Perityphlitis and leitet die, ,übliche" Behandlung ein. Der Zustand des Kindes verschlimmerte sich zusehends, namentlich trat völlige Stuhl- und Windverhaltung ein, und zudem erbrach Patientin alles, was sie $\mathrm{zu}$ sich nahm. Am I2. VI. stellt der behandelnde Arzt Peritonitis fest und schickt das Kind in das Spital.

$\mathrm{S}$ t a t u s: Kind sieht blaß und verfallen aus; Facies abdominalis; leichte Trübung des Sensoriums. Abdomen sehr stark aufgetrieben, Darmzeichnung, Bauchhaut livide verfärbt. Ganze Unterbauchregion schallt gedämpft; Palpation unmöglich. Im Rektum viel Kot; $m$ a $n$ fühlt extrarektal nach rechts und median einen Tu mor.

Operation: Zunächst Schnitt parallel dem Ligamentum Pouparti, unter der Diagnose Perityphlitis mit Abszeßbildung und Peritonitis progrediens. In der Bauchhöhle ke in Ex su dat. Rektifikation der Diagnose auf Invagination; bogenförmige Erweiterung des Schnittes. Invaginatio ,ileo-coecalis“. Dünndärme außerordentlich stark gebläht. Desinvagination gelingt nicht. Es muß die R e s e $\mathrm{k} t \mathrm{i}$ o $\mathrm{n}$ ausgeführt werden.

Das Kind erholt sich nicht aus seinem schweren Kollaps, und noch am Abend des Operationstages, um ro $\frac{1}{2} \mathrm{Uhr}$, tritt $\mathrm{E} \times$ it us ein.

A u topsie: Reselition des Cöcum sowie der untersten Partie des Ileum, Endverschluß, seitliche Inplantation des Ileam in das Colon transversum. Akuter Katarrh des untersten Ileumabschnittes (?), Schwellung der Mesenterialdrüsen. Die Nahtstellen in gutem Zustande, keine Peritonitis. Aspiration von Darminhalt. Trübe Schwellung des Myokards, Verfettung von Leber und Nieren.

5. Charles J. 4 Mo nate. Eintritt I8. XII. Igog.

Am I3. XII. begann das Kind zu erbrechen und weinte viel; seither Stuhl- und Windverhaltung. Klystiere und Kalomelgaben hatten nur Abgang von Schleim und Blut zur Folge. Während am 15. und I6. XII. noch mehrmals heftiges Erbrechen auftrat, hat Patient heute nicht mehr erbrochen. Kein Fieber.

$\mathrm{S} t$ a $t \mathrm{us}$ : Patient ist benommen und hat einen frequenten, schlecht gespannten Puls. Abdomen nicht gespannt. In der Fossa iliaca sinistra fühlt man eine rundliche, oblonge Geschwulst, die jedenfalls einer Invagination entspricht. Per rectum fühlt man im Darm eine Geschwulst mit trichterförmiger Offnung in de $\mathrm{r}$ M i t t e; beim Pressen tritt dieser Tumor im Analring zutage. Versuche, mit Hilfe einer Sonde zu desinvaginieren, mißlingen.

Operation: Medianschnitt vom Nabel zur Symphyse. Es findet sich eine doppelte Invagination: das Sigmoideum 
ist in das Rektum invaginiert, während das Ileum eine ganz kurze Strecke weit in das Cöcum, dieses mit dem Ileum analwärts durch das Colon ascendens und transversum hindurch - diese mit sich invaginierend in das Colon ascendens und den Anfangsteil des Sigmoideum eingestülpt ist. Die Invagination des Sigmoideum läßt sich mühlelos reduzieren, dann wird durch Zug am Ileum die Coloninvagination gelöst, gelingt aber nicht vollständig. Der letzte Teil des Cöcum mit dem Appendix läßt sich nicht mehr entwickeln. Die Kuppe des Processus vermiformis ragt aus dem äußeren Zylinder heraus. Man sieht sich zur Resektion gezwungen, verschließt beide Darmenden und legt eine Seite-zu-SeiteAnastomose an.

Ver la uf: Vom I9. zum 20. XII. treten Stuhl und Winde auf; Abdomen weich und nicht druckempfindlich. Am Nachmittag des 20. XII. Krämpfe, Nahrungsverweigerung. In der Nacht zum 2I. XII. Exitus, bei antemortaler Temperatur von $4 \mathrm{I}^{\circ}$.

A u topsie: Nahtstelle schön, Naht hat gehalten. Keine Peritonitis; Därme enthalten nur wenig Gas. Zwischen den Mesenterialblättern an der Resektionsstelle kleine Hämatome. Anastomose genügend weit. Die Partie des Colon sigmoideum, welche die Spitze der tiefen Invagination bildete (die sich leicht lösen ließ), sieht nicht gut aus. Der Darm ist mißfarbig und infiltriert an entsprechender Stelle, zeigt jedoch keine Serosadefekte und keine Perforation. D e r a ufgeschnittene Darm zeigt an dieser Stelle a usgedehnte hämorrhagische Infarcierung der Schleimhaut. Ein Bezirk von $2: 3 \mathrm{~cm}$ ist nekro$\mathrm{t}$ is ch und ulceriert. Rings um das Geschwür zahlreiche umschriebene Hämorrhagien. Trübe Schwellung des Myokards. Übrige Organe normal.

Das kleine Material kann uns natürlich nicht Anlaß zu irgendwelchen statistischen Deduktionen geben, seine Verwertung in Form einer klinischen Besprechung als Beitrag zur Invaginationslehre dürfte sich immerhin rechtfertigen. Bei dieser Gelegenheit möchte ich übrigens darauf aufmerksam machen, wie wenig zuverlässige Resultate die Statistik gerade in der Frage der Darminvagination uns bietet, weil die Angaben über prozentuale Heilungen mit blutigem oder unblutigem Verfahren die verschiedensten Fälle promiskue zusammenfassen. Nun ist doch zwischen einer ganz frischen Invagination, bei der höchstens leichte Stauung und Hyperämie am Kopfe des Invaginatums bestehen, und einem Falle, der schon alle sekundären Konsequenzen der Mesenterialstrangulation darbietet, gewiß ein so gewaltiger Unterschied, daß beide Fälle eine ganz separate Betrachtung erfordern. Leider 
spielen aber kritiklos akzeptierte Statistiken auch heute noch in der Diskussion über das empfehlenswerteste therapeutische Verfahren eine große Rolle. Wir werden die Frage bei Besprechung der Therapie noch $\mathrm{zu}$ streifen haben.

Wie gewöhnlich, überwiegt auch bei unserer kleinen Gruppe das männliche Geschlecht, indem 4 von den 5 Patienten Knaben sind; zudem stehen diese 4 Kinder im ersten Lebensjahre, während das Mädchen $2 \frac{1}{2}$ Jahr alt war. Auch die Altersverteilung entspricht somit bekannten Verhältnissen. - Hinsichtlich der A n a m $\mathrm{n}$ e s e interessieren die $\mathrm{D}$ a $\mathrm{u}$ e $\mathrm{r}$, sowie die vor Spitaleintritt eventuell beobachteten pat hog nom on is chen Symptome, als welche die Symptome des plötzlich, eventuell nach vorgängigen Darmstörungen, einsetzenden Ileus, mit Erbrechen, Obstipation und Abgang von blutigem Schleim, sowie der eventuelle Nachweis eines Tumors durch den zuweisenden Arzt in Frage kommen. Eigentlichen $\mathrm{Fr}$ ühfall verzeichnen wir keinen, wenn wir nicht $\mathrm{Nr}$. I als solchen betrachten, bei dem die ersten Symptome, schmerzhafte Krämpfe und blutig-schleimige Dejektionen, 36 Stunden zurück lagen. Alle anderen Patienten kamen spät in Behandlung, da sie erst 2, 3, 5 und 7 Tage nach Beginn der Erkrankung in das Spital eingewiesen wurden.

Vorausgehende Verdaungsstörungen, die für die Auslösung der Invagination in Betracht kommen konnten, zeigte nur Nr. 2; die anderen Patienten waren aus vollem Wohlbefinden heraus plötzlich erkrankt. Ganz illustrativ ist die Angabe bei Fall Nr. 3, daß der Úbergang von der Brusternährung zur künstlichen Ernährung die E rkrankung a uslöste. Da unverständigerweise dem Kinde gleich Milch mit eingeweichtem Brot verabfolgt wurde, begreift man, daß die Peristaltik sich dieser ungewohnten Aufgabe gegenüber unphysiologisch verhielt. Der B e g i n $\mathrm{n}$ war in allen Fällen, abgesehen von $\mathrm{Nr}$. 2, ein ganz akuter, mit heftigem $\mathrm{Sch}$ m e r za $\mathrm{f}$ a 11 , auf den erst am zweiten Tage Erbrechen folgte (Nr. I u. 4) oder direkt mit Erbrechen (3 u. 5); man ist wohl berechtigt, das initiale Erbrechen als reflektorisches, das sekundär einsetzende als Folge des Darmverschlusses ( $\mathrm{m}$ e $\mathrm{ch}$ a $\mathrm{n}$ is che $\mathrm{s}$ im weiteren Sinne) zu betrachten. - Der Verlauf der Invagination bei Patient Nr. 2 war mehr ein chronischer oder subakuter, 
insofern, als während mehreren Tagen Symptome bestanden, die irrtümlicherweise auf einen linksseitigen Abszeß mit periodischer Entleerung in den Darm bezogen wurden (Abgang von Blut mit Schleim und Eiter(?)), während erst am sechsten Krankheitstage plötzlich Erbrechen und Stuhlverhaltung einsetzten. Diese plötzliche Änderung im klinischen Bilde hat ihre Ursachen wohl im Hinzutreten intensiver Strangulation oder darin, daß ein vorher noch permeables Hindernis infolge der sekundär am Invaginatum in Erscheinung tretenden Veränderungen undurchgängig wird. Alle Patienten hatten, entsprechend dem bestehenden DarmverschluB, a $\mathrm{n}$ a mest is $\mathrm{ch}$ Stuhlverhaltung zu verzeichnen; allerdings war diese Obstipation keine absolute, insofern als sich der unterhalb der Invaginationsstelle befindliche Darm noch entleerte, z. T. auf Klysmen (Nr. 3 u. 5) oder Calomelgaben: Wiederholung dieser evakuierenden Ma Bnahmen hattekeinen Erfolg, führtedagegen $\mathrm{zuEntle}$ erung von Blutund blutigem Schleim. Der für Invaginationen charakteristische Blutabgang wurde nur bei Nr. 4 vermißt, ein Fall, der ganz unter den Symptomen der perforativen Appendicitis verlief und auch unter dieser Diagnose operiert wurde. - Bei Patient Nr. 2 traten anfänglich D i a r $\mathrm{rhöen}$ auf, wohl ein Symptom der auslösenden akuten Verdauungsstörung infolge des Nahrungswechsels. Der $\mathrm{N}$ a $\mathrm{ch}$ we is e in es $\mathrm{T} \mathrm{u}$ mors war vor der Einweisung bei Nr. 3 und auch bei Nr. 2 geglückt, hatte aber in letzterem Falle zu der irrtümlichen Annahme eines Abszesses geführt.

Hinsichtlich Status und Diagnose interessieren in vorliegendem Material folgende Einzelheiten: Der erste Fall bot einige Anhaltspunkte für die Annahme einer Invagination, insofern, als leichte Auftreibung, das Vorhandensein von blutigem Schleim im Rektum und die relative Leerheit der Fossa ileo-coecalis (Dancesches Symptom) im Zusammenhalt mit der Anamnese die Annahme einer Darmeinscheidung höchst wahrscheinlich machten. Immerhin gelang der Nachweis eines I n $\mathrm{vag}$ inationstumors auch in Narkose trotz sorgfältiger Palpation nicht, und es bedurfte deshalb eines gewissen diagnostischen Entschlusses, um trotzdem eine Intussuszeption anzunehmen. 
Wenn man eine Invagination nur diagnostizieren will, wenn alle klassischen Symptome, also auch der Nachweis eines Tumors, vorhanden sind, dann wird man eine Anzahl Fälle notwendigerweise verlieren. Nach Leichtenstern gelingt ja der Nachweis eines Tumors nur in 5o Proz. der Fälle, nach T r e v e s in 6r Proz., während allerdings $\mathrm{H}$ an se $\mathbf{n}$ unter den 28 Fällen der Tscherningschen Abteilung nur zweimal die Invaginationsgeschwulst nicht palpieren konnte. Wo bei zutreffender Anamnese im Status nur der palpable Tumor fehlt und dieses Fehlen einer Geschwulst, wie in unserem ersten Falle, sich zudem noch erklären läßt - positives Dancesches Symptom, dem W il m s allerdings nur theoretische, keine praktische Bedeutung zuzusprechen geneigt scheint - wird man sich in der Diagnose nicht irre machen lassen. Bei unserem zweiten Patienten war der Tumor fälschlicherweise für einen linksseitigen Abszeß gehalten worden, was sich aus dem subakuten Verlauf der Intussuszeption erklärt. Immerhin stellte der Chirurg sofort die richtige Diagnose, sobald die akuten Erscheinungen einen bestimmten Hinweis boten. Im dritten Falle war bei Spitalantritt die Auftreibung so hochgradig geworden, daß der früher konstatierte Tumor sich dem Nachweise entzog; auch bei Nr. 4 fehlte eine palpatorisch nachweisbare Geschwulst bei Untersuchung von außen, während die Rektalexploration einen Tumor rechts und median ergab, der als perityphlitischer Absze $B$ angesprochen wurde. Der letzte Patient endlich zeigte einen typischen Invaginationstumor, allerdings in ungewöhnlicher Weise in der linken Fossa iliaca lokalisiert. Der B ef und per rectum war für Invagination entscheidend in einem Falle (Nr. 5), wo man das Invaginatum fühlte und beim Pressen in der Analöffnung sehen konnte; bei Nr. 2, 3 und 4 gelang der Nachweis einer dem Tumor entsprechenden Resistenz durch die Rektalwand hindurch. Doch hat dieser Nachweis für die Diagnose nur beschränkten Wert, wenn die Geschwulst nicht schon von außen palpabel ist, wie ja unser vierter Fall zur Evidenz beweist.

Gestützt auf Anamnese und Status, gelang die Diagnose in allen Fällen mit Ausnahme von Nr. 4, der als Appendizitis mit AbszeBbildung und beginnender diffuser Peritonitis zur Operation kam. Es handelte sich eben um einen der fortgeschrittenen Fälle, 
bei denen die Differentialdiagnose zwischen Ileus und Peritonitis nicht mehr gestellt werden kann. Hochgradiger $\mathrm{M}$ e $\mathrm{t}$ e o $\mathrm{r}$ is $\mathrm{m}$ u s bestand nur bei Nr. 3 und 4 ; die beiden Fälle boten, wie übrigens auch Nr. I und 2, deutliche Darm z e i chn ung und lebhafte $\mathrm{P}$ e r is t a $1 \mathrm{t}$ i k im zuführenden Dünndarm. Koterbrechen und Tenesmus wurden nie konstatiert.

Úber das o perative Verf a hre n können wir uns kurz fassen: In zwei Fällen, bei Nr. I und 3, gelang die D e s v a g i n a t i o $n$, während in den übrigen Fällen die $\mathrm{Resektion}$ ausgeführt werden mußte. Patient Nr. 5 zeigte eine doppelte, also fünfzylindrische Einscheidung; hier konnte die äußere, offenbar später entstandene Invagination des Dickdarms reponiert werden, während die Reduktion der ,,ileo-coecalen" Einstülpung nicht gelang. Im zweiten Falle wurde zunächst ein Versuch mit dem von Rydygier namentlich empfohlenen Jesset-Barkerschen Verfahren (Resektion des Invaginatum allein) gemacht, der schlechten Ernährung des eintretenden Ileums wegen jedoch aufgegeben. Wir können die Vorteile dieser Methode vor einer glatten Resektion nicht recht einsehen. - Die Resektion war in unseren Fällen durch absolute Unmöglichkeit der Desinvagination gegeben; entsprechend den weit vorgeschrittenen Veränderungen am Invaginatum handelte es sich um längere Dauer der Intussuszeption (Nr. 2 hatte $4-5$ Tage, der vierte und fünfte Fall hatten 7 resp. 5 Tage gedauert). Die Grenze für die Möglichkeit operativer Desinvagination bildete das Ende des zweiten Tages. Über die besondere Art des Resektionsverfahrens und der Desinvagination ist nichts vom Gewöhnlichen Abweichendes zu sagen. Wir möchten nur erwähnen, daß uns der Zug am Invaginatum zur Unterstützung des Druckes von außen auf den Kopf des Intussuszeptums sehr wertvolle Dienste zu leisten scheint; Zug allein halten wir mit $\mathrm{Kl}$ e $\mathrm{m} \mathrm{m}$ für zu wenig wirksam und für gefährlich. Wo das Mißverhältnis zwischen der Weite des Halses und dem Durchmesser des Kopfes der Invagination zu groß geworden ist, hat jede Forcierung der Taxis keinen Sinn und kann dem Patienten nur Schaden bringen. Meistens gelingt es sehr rasch, sich davon zu überzeugen, ob eine schonende Desinvagination noch möglich ist oder nicht.

Größeres Interesse als das operative Vorgehen bietet $\mathrm{der}$ intra operationem erhobene Befund in unseren 
Fällen, der uns zu einigen prinzipiellen Auseinandersetzungen Anlaß gibt. Nach übereinstimmendem Urteil der Autoren ist die häufigste Invaginationsform bei Kindern die sogenannte Invaginatio ileocoecalis, die $z$. B. nach der Zusammenstellung von $W$ e i $B$ bis zum Pubertätsalter $42-43$ Proz. ausmacht. Nun herrscht aber in der Anwendung dieser Bezeichnung eine gewisse Konfusion, die es unmöglich macht, die in den Krankengeschichten vorkommende Bezeichnung, ,ileocoecalis" ohne weiteres zu verwerten. Das geht schon aus der Tatsache hervor, daß L e i c h t e n stern die drei Abarten der Ileocöcalis, ileo-colica und iliacaileocolica unterscheidet. Dabei versteht er, wie auch Wilms und andere Autoren unter der Inv. ileocoecalis im engeren Sinne diejenige Form, bei welcher das ileocöcale Ostium, die Valvula Bauhini, vorangeht. Bei der Inv. ileocolica soll zunächst ein Prolaps der Mucosa des untersten Ileums in das Colon stattfinden, während bei der iliaca-ileocolica eine primär im untersten Ileum entstandene Invagination sich sekundär durch die Cöcalklappe in das Colon einstülpt. W il m s kritisiert die Annahme eines Schleimhautprolapses in das Colon in Parallele zum Prolapsus recti wegen der fehlenden Druckdifferenz und macht darauf aufmerksam, daß man aus einer fertigen Invaginatio ileo-colica mit sekundärer Cöcum- und Coloninvagination nicht mehr erkennen kann, ob das Einschieben des Ileums ins Colon resp. Cöcum im Sinne eines Prolapses oder im Sinne einer echten, primären Invaginatio iliaca mit konsekutiver Umstülpung des untersten Ileums zustande gekommen ist. Deshalb schlägt W i l m s vor, nur von einer Invaginatio ileocöcalis $\mathrm{zu}$ sprechen und den Begriff der ileocolica auszuschalten. Im übrigen scheint es $W$ i $1 \mathrm{~m} \mathrm{~s}$ am empfehlenswertesten zu sein, die Bezeichnung der Invagination nach dem Teil zu wählen, der bei der beginnenden Einscheidung die Spitze bildet, was uns gestattet, neben einer Iliaca und Colica eine Ileocöcalis anzunehmen, bei welcher die Klappe der Art der ersten Einstülpung ist. Die Invaginatio iliaca-ileocolica bleibt natürlich von diesem Gesichtspunkte aus eine Iliaca. Wir werden diese Vorschläge weiter unten diskutieren und weisen an dieser Stelle nur darauf hin, daß die Unklarheiten im Begriff der Invaginatio ileocoecalis eine genauere Verwertung dieser Angabe in den Krankengeschichten, wie schon erwähnt, nicht gestatten. 
Es unterliegt keinem Zweifel, daß unter dem Begriff der Ileocöcalis eine ganze Reihe von sog. ileocolischen oder iliaca-ileocolischen Einscheidungen figurieren. $\mathrm{D}$ as $\mathrm{zeigt} \mathrm{schon} u \mathrm{nser}$ kleines Material, bei welchem $z$ wei Fälle als Invaginationesileocoecales rubriziert sind, während der Operateur, gemäß der beigelegten Zeichnung, iliaca-ileocolische Invaginationen mit sekundärerEinstülpungdes Colonsund Nach-

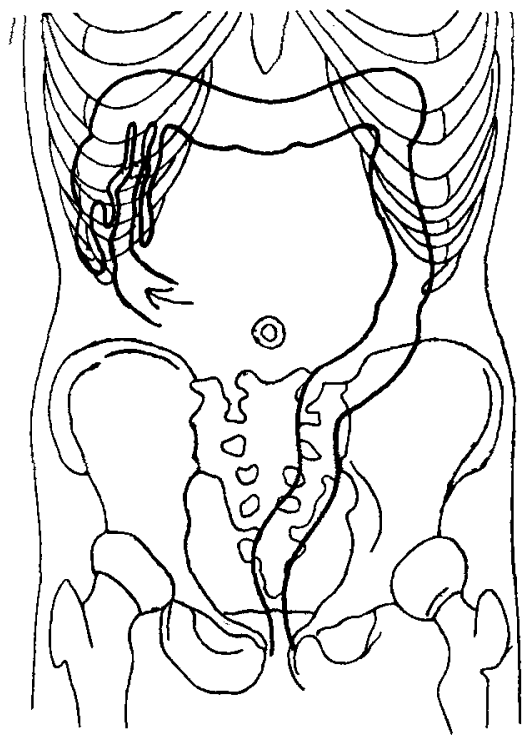

Fig. I. Schematische Darstellung der Invaginationen bei Fall I, 2. 3 und 4. (Invag. ileocoeco-colica unsrer Nomenklatur.)

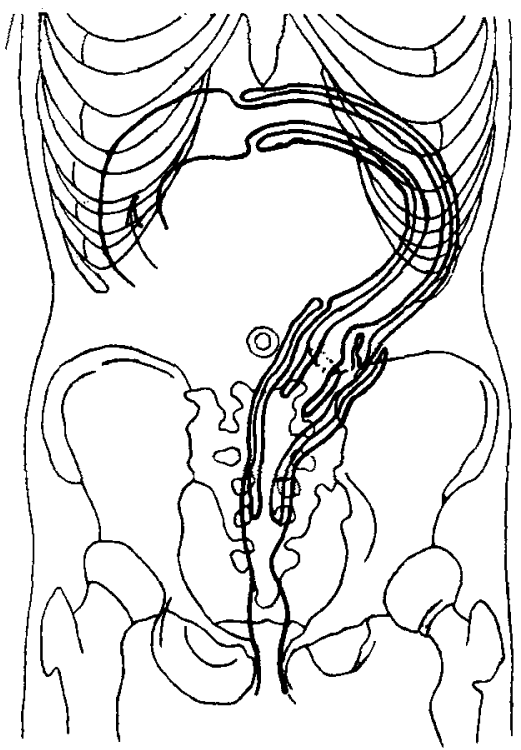

Fig. 2. Schematische Darstelluug der Invagination bei Fall 5. (Invag. ileocoeco-colica und Invag. sigmoideo-rectalis, duplex.)

zi ehen des Cöcums festgestellt hat te. - Diese Einscheidungsform boten unsere ersten vier Fälle dar, während der fünfte eine Kombination dieser Form mit einer Invagination des Sigmoideum in das Rektum darstellte. Es handelte sich also um die seltene doppelte oder fünfzylindrische Einscheidung. Um jedem Mißverständnis vorzubeugen, geben wir von jedem der beiden Invaginationstypen eine schematische Zeichnung (Fig. I und 2). Im ersten Falle interessiert das Vorhandensein einer Bride, die zur Täuschung über die Mechanik des Ileus hätte Anlaß geben, 
können, bei dem schwierigen Nachweis des Tumors, sowie eine Torsion des Appendix und Verwachsung desselben mit dem Mesocöcum. Vielleicht war dieses Verhalten ätiologisch von Bedeutung. Bei dem zweiten Fall erwies sich der invaginierte Dünndarm als torquiert; entsprechend waren am Hals des Invaginatum intensive Ernährungsstörungen zu konstatieren.- Die Bauchwunde wurde stets ohne Drainage primär geschlossen.

Der postoperative Verla uf war bei Nr. I und 3, wo die Desinvagination glückte, ein günstiger, bei den anderen, der Resektion unterworfenen Fällen ein letaler. Wir dürfen daraus nicht ohne weiteres schließen, daß die Resektion so ungemein viel gefährlicher sei, da ja der schlimme Ausgang wesentlich durch die längere Dauer des Darmverschlusses in diesen Fällen gegeben war. Aber wir sehen die Tatsache bestätigt, daß Kinder, wenn sie schon unter dem Einflusse längerdauernder Intoxikation vom Darme her mit der schon durch die Zirkulationsverhältnisse beim Ileus gegebenen mißlichen Rückwirkung auf den Blutdruck stehen, den Eingriff der Resektion sehr schwer überstehen. Schon die Eventration der Därme an sich bedeutet ja oft in diesen Fällen ein gewagtes Unternehmen. I ch g la $u$ be desha $1 \mathrm{~b}, \mathrm{da} \boldsymbol{B} \mathrm{die}$ statistischen Zahlen der Resektionsresultate, wie sie in den groBen Statistiken enthalten sind, kein zutreffendes Bild von der Gefahr der Darmresektion geben, weil offenbar die meisten letal verlaufenen Fälle nicht publiziert werden -wenigstens vereinzelte Fälle, nicht solche innerhalb gröBerer oder kleinerer Serien - und ferner, weil wohl auch von einzelnen Chirurgen früh zur Operation eingelieferte Fälle reseziert werden konnten. Die Prognose dieser relativen Frühfälle stellt sich natürlich ganz anders dar.

Im Verlaufe der zwei geheilten Fälle ist die anfängliche, während $\mathrm{I}-2$ Tagen bestehende gan $\mathrm{z}$ erhebliche Te mperatursteigerung bis zu $39^{\circ}$ und $39,7^{\circ}$ (Fall I und 3) sehr beachtenswert. Wir wissen zwar, daß viele Kinder auch nach relativ einfachen Operationen und aseptischem Wundverlauf vorübergehend recht hohe Temperaturen aufweisen. Wir haben das namentlich bei Herniotomien und Hydrocelenoperationen zu beobachten Gelegenheit gehabt. Wo in solchen Fällen eine Äther- 
wirkung auf die Lungen ausgeschlossen werden kann, bildet die Annahme einer Resorption im Wundgebiete die plausibelste Erklärung. Doch gehören diese postoperativen Temperaturanstiege nicht zu den Regelmäßigkeiten und fehlen namentlich dort, wo exakte Blutstillung möglich war. Die Erklärung für diese Temperutursteigerungen ist wohl in folgendem zu suchen: Durch die Invagination wird das Mesenterium des Invaginatums stranguliert; es kommt zu einer venösen Stase mit multipler Thrombenbildung. Gleichzeitig treten am zugehörigen Darm die Folgen der Ernährungsstörung in Form von Odem, hämorrhagischer Infarzierung und von der Schleimhaut zur Serosa fortschreitender Nekrose auf. Schleimhautnekrosen treten schon nach relativ kurzer Dauer der Einklemmung in Erscheinung, und damit ist auch dem Eindringen der Darmbakterien in die Darmwand der Weg eröffnet. Wird nun desinvaginiert, so wird der Rückfluß des venösen Blutes wieder frei; es kommt sogar infolge der Erhöhung des arteriellen Druckes in den betreffenden Zirkulationsgebieten zu einer direkten Ausschwemmung der in die Darmwand eingedrungenen Bakterien und ihrer Toxine. Zugleich gelangen auch die toxischen Stoffe aus den nekrotischen Darmwandpartien in die Zirkulation. Diese Einschwemmung von Toxinen und Bakterien ist der Grund der hohen postoperativen Temperatursteigerung, und die Erscheinung verdient alle Beachtung, da sie das Schicksal des Patienten noch nachträglich in schlimmem Sinne entscheiden $\mathrm{k}$ a n n. Einen Beweis für die Richtigkeit dieser in der Literatur nicht berücksichtigten. Tatsache haben wir in unserem letzten Falle. Hier war die äußere Einscheidung, die Invagination des Sigmoideums in das Rektum, bei der Operation reduziert worden, während Ende des Ileums sowie Cöcum und Anfang des Colon ascendens reseziert werden mußten. Patient starb am dritten Tage nach der Operation, obschon die Durchgängigkeit des Darmes schon vom zweiten Tage ab wieder hergestellt war. Der Exitus erfolgte unter Krämpfen und Temperaturanstieg a uf $4 \mathrm{I}^{\circ}$ !, Symptome, die unbedingt für eine Intoxikation sprachen. Die Autopsie zeigte nun den Ausgangspunkt der Resorption in einer Darmwandnekrose, welche dem Kopf, d. h. der Umschlag- 
stelle der sigmoidealen Invagination entsprach. Auch in der Kusuistik K le m m s finden wir einen ähnlichen Fall verzeichnet, bei dem allerdings gleichzeitig noch bronchopneumonische Herde vorlagen. Es ist jedenfalls naheliegend, anzunehmen, daß die nach Desinvagination auftretenden Pneumonien oft embolischer Natur sind. Natürlich kanndurchrohes Manipulieren bei der Desinvagination die Resorption septischer Stoffe wesentlich gefördertwerden, und da B diese Möglichkeit gegen die Anwendunglangdauernderforcierter Desinvaginationsversuche - blutiger oder unblutigerspricht, liegt für uns auf der Hand.

Zum Mechanismus der Invaginationen im allgemeinen läßt sich aus unserem Material nicht viel Beweisendes ableiten; immerhin möchten wir erwähnen, daß im Verhalten des torquierten und mit dem Mesocöcum ausgedehnt verwachsenen Appendix im Falle Nr. I möglicherweise eine anatomische, prädisponierende Ursache lag. Wir stellten uns das so vor, daß durch diese Verwachsung des Appendix eine gewisse Fixation des ileocöcalen Ostiums zustande kam, welche für die Peristaltik, d. h. für den Ablauf der peristaltischen Welle, ein gewisses Hindernis darstellte. Solche Widerstandsvermehrungen haben erfahrungsgemäß eine Steigerung der Peristaltik zur Folge und können, namentlich wenn die Peristaltik unregelmäßig wird, zur Bildung von Intussuszeptionen führen. - Der Nahrungswechsel, welcher nach $H$ or $n$ bei Säuglingen für viele Einscheidungen verantwortlich zu machen ist, hatte bei unserem dritten Patienten unmittelbar vor dem Auftreten der Invagination stattgefunden.

Bestimmtere $\mathrm{Hinw}$ is e bieten unsere Krankengeschichten hinsichtlich des Ortes, wo die pri$\mathrm{märe} I \mathrm{n} v a \mathrm{~g}$ in a tio $\mathrm{n}$ bei sog. ileocöcalen Invaginationen $s t a t t f i n d e t$, eine Frage, deren Discussion noch offen steht, und ohne deren Entscheidung eine maßgebende Erklärung des Mechanismus der ileocöcalen Intussuszeptionen sich nicht geben läßt. Bekanntlich hat man die Häufigkeit der Invaginatio ileocoecalis beim Kinde mit der großen Lumendifferenz des Dünn- und Dickdarmes in Zusammenhang gebracht, indem man darauf hinwies, daß bei der Geburt die Weite von Colon und Ileum beinahe 
dieselbe ist, da $B$ dann aber das Colon bedeutend rascher wächst und sein Lumen sich im Laufe von 4-6 Monaten verdreifacht, oder vervierfacht, während dasjenige des Ileums sich in der gleichen Zeit nur etwa verdoppelt. D'A r c y - P ow e r glaubt nun, daß infolge dieses raschen und ungleichmäßigen Wachstums die Ileocöcalklappe schlußunfähig werde, eine Annahme, der die Befunde $\mathrm{Hirschsprungs}$ zu widersprechen scheinen. DaB eine weitergehende ileocöcale Einschiebung nur stattfinden kann, wenn ein relativ langes Mesenterium des Cöcums oder Colons vorhanden ist, geht aus einer einfachen Uberlegung hervor. W i $1 \mathrm{~m} \mathrm{~s}$ hat nun darauf aufmerksam gemacht, da $B$ in der Literatur die Tatsache zu wenig berücksichtigt ist, auf die wir schon oben hingewiesen haben, daß nämlich ein Teil der sog. ileocöcalen Einscheidungen aus primären reinen Iliacalinvaginationen hervorgegangen sein können, die kurz vor der Klappe entstehen und zunächst zu ileocolischen Einscheidungen werden, unter allmählichem Nachziehen des Cöcums zu sog. ileocöcalen. Geht nur ein kurzes Stück Ileum durch die Klappe, (primär) so ist nachträglich schwer zu entscheiden, ob wirklich die Bauhinsche Klappe vorangeht oder nicht. Daß eine fertige Invaginatio ileocolica aus einer Iliaca hervorgehen kann, beweist zunächst unser erster Fall, bei dem die Stelle der primären Invagination im untersten Ileum sich mit aller wünschbaren Sicherheit feststellen ließ. Nun sind merkwürdigerweise alle unsere fünf Fälle sog. ileocöcaler Invaginationen aus derartigen primären Iliacalintussuszeptionen hervorgegangen. Wir fügen eine schematische Darstellung der Entwicklung dieser (aus primären Iliacaleinstülpungen hervorgegangenen) ,ileocöcalen“ Invaginationen bei (Fig. 3-6). H a n s e $n$ berichtet über drei solche Fälle, $\mathrm{Kl}$ e $\mathrm{m}$ m über eine analoge Beobachtung, die er irrtümlicherweise für eine doppelte Einschiebung hält (Nr. 8); L e i ch te n ster $\mathrm{n}$ hat in seiner umfassenden Arbeit nur vier Beispiele dieser Art. Diese spezielle Form wäre somit eine Seltenheit, wenn nicht die Wahrscheinlichkeit bestünde, daß eine große Anzahl der mitgeteilten ,,ileocöcalen“ Invaginationen solche primäre Iliacaleinstülpungen waren. Mit Sicherheit läßt sich diese Annahme nachträglich nicht mehr entscheiden, weil eben die zum Teil konfuse Nomenklatur die Angaben der Autoren in den Krankengeschichten unbrauchbar macht. 
Dagegen spricht verschiedenes dafür, daß die noch heute so ziemlich allgemein geltende Anschauung, nach der die Ileocöcalregion ganz bestimmte Dispositionen für die Entstehung von Darminvaginationen darbiete, in der bisher vorgetragenen Form nicht zutrifft. Zunächst sei darauf hingewiesen, daß die bekannte große Lumendifferenz zwischen Ileum und Cöcum gerade für die von der Ileocöcalinvagination am meisten heimgesuchte Periode des

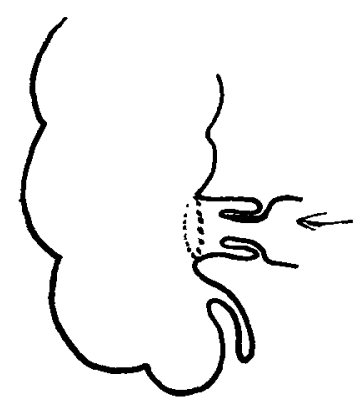

Fig. 3.

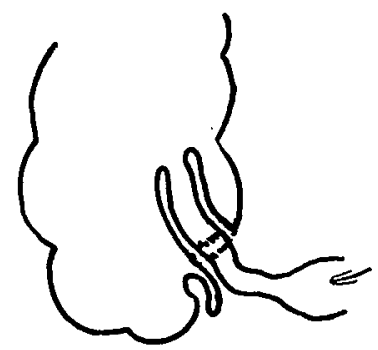

Fig. 5 .

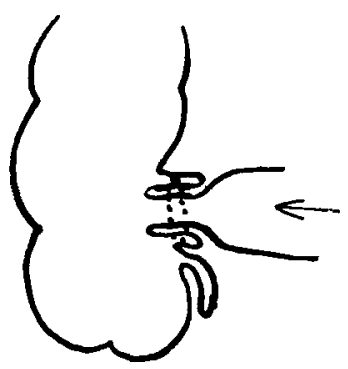

Fig. 4.

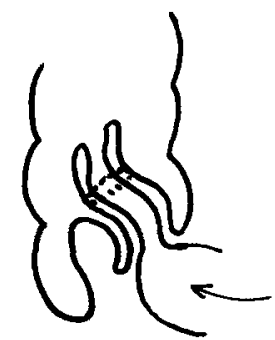

Fig. 6.

Fig. $3,4,5,6$. Schematische Darstellung der Entwicklung, ,ileocoecaler" resp. ,ileocoeco-colischer" Invaginationen aus einer primären Iliacaleinstülpung.

Säuglingsalters am wenigsten in Betracht kommt, in dem sich der große Unterschied in der Lichtung der beiden Darmpartien crst bis zum fünften Lebensjahre voll entwickelt. Die von $\mathrm{H}$ e r z angenommene Insuffizienz der Ileocöcalklappe ist, wie erwähnt, durch die Befunde $\mathrm{H}$ irs chs prungs widerlegt, der auf diesen Punkt besondere Aufmerksamkeit verwendete; gegenüber I, e i c h t e nsterns Auffassung, nach welcher an der Ileocöcalklappe ein Schleimhautprolaps des Ileums entstehe, analog der Bildung eines Prolapsus recti, macht $W$ i 1 m s geltend, daß von einer Druck- 
differenz zwischen Ileum und Cöcum die Rede nicht sein könne, während für den Rektalprolaps in letzter Linie der Unterschied zwischen der Bauchpresse und dem äußeren Luftdruck mechanisch in Betracht kommt. So bleibt von den vielen disponierenden Momenten nur das lange Mesenterium oder das Mesenterium commune, ein anatomisches Verhalten, das ebensogut eine primäre Iliacaleinstülpung $\mathrm{zu}$ einer sekundären ileocolischen werden läßt, als es eine primäre Einstülpung des Cöcums mit passivem Nachziehen des Ileums erlaubt. Gleich verhält es sich mit dem prädisponierenden fettarmen Mesenterium. Es zeigt sich also, daB man a u älteren Bearbeitungen die Lehrevon der primären Einstülpung der Bauhinschen Klappe und die für den speziellen $Z$ weck konstruierte Lehre von einer speziellen anatomischen Disposition, welche das Uberwiegen einer Invaginationsform zu erklären geeignet erscheint, wie es so oft geht, ziemlich kritiklos a uch in neuere Bearbeitungen des Themas aufgenommen hat. Wenn schon die Unzuverlässigkeit der Nomenklatur und die Haltlosigkeit der Dispositionsargumente unsere Zweifel erweckten, so bekamen diese Zweifel eine festere Basis durch unsere übereinstimmenden Befunde primärer Iliacalinvagination, welche zu sekundären ileocolischen und ileocöcalen Einstülpungen geführt hatten; in letzter Zeit erschienen nun zwei Publikationen, die einen energischen Angriff auf die alte Lehre von der Ilocöcalinvagination bedeuten.

B l a u e ${ }^{1}$ ) berichtet über drei von ihm operierte Fälle, bei denen er nach seiner Auffassung mit Sicherheit den Ort der ersten Einstülpung an der Kuppe des Cöcums nachweisen konnte; diese war in allen Fällen ödematös infiltriert, in einem Falle sogar fibrinös belegt und verdünnt, dazu stets trichterförmig eingezogen. Beweisend erscheint namentlich der dritte Fall, in welchem die Ileocöcalklappe gar nicht an der Einstülpung beteiligt war, sondern nur die Cöcalkuppe mit der Basis des Appendix $5 \mathrm{~cm}$ weit in das Colon ascendens eingestülpt sich erwies. Dazu gelang nach dem Autor in allen Fällen der Nachweis, daß die veränderte Partie des Cöcums, und keineswegs die Klappe an der Spitze der Invagination marschierte. Unter Akzeptierung des Nothnagel-

I) Beiträge zur klin. Chirurgie I9I0, Bd. 68, Heft I. 
schen Satzes, daß dasjenige Darmsegment, welches den ursprünglichen Ausgangspunkt der Invagination gebildet hat, auch an der Spitze derselben bleibt, nimmt $\mathrm{Bl}$ a u el an, daß in seinen Fällen die Kuppe des Cöcums den Ort der ersten Entscheidung bildete. Der Autor fand dann noch drei analoge Resektionspräparate und hat an Hand von Literaturstudien die Ủberzeugung gewonnen, daß der cöcale Entstehungstypus bei der reinen Invaginatio ileo-coecalis die Regel bilden müsse. Zunächst fand er in der Literatur mehrfach reine Cöcalinvaginationen, wie sie seinem dritten Fall entsprechen, und widerspricht der Auffassung von Wilms, der für diese Form Adhäsionen und pathologische Prozesse verantwortlich macht. Auch bei der ausgebildeten Invagination ist der Vorantritt des Cöcums beschrieben. So kam L ore nz, der ebenfalls drei Fälle analog den von Bl a u el publizierten behandelte, $z \mathfrak{u}$ der schon 1905 von ihm niedergelegten Anschauung, daß in der großen Mehrzahl der Fälle ileocöcaler Einstülpung die Kuppe des Cöcums der Ort der primären Invagination sei, weshalb er die Bezeichnung c o e co-coli c a vorschlägt. Ferner hat Deville $\mathrm{chab}$ rolle über 32 appendicocöcale Einscheidungen berichtet, die bei der passiven Rolle der Appendixeinstülpungen - abgesehen von den Fällen, wo sich die abnorm weite Basis primär einstülpt - wesentlich als Bereicherung der Kasuistik cöcaler Einscheidungen zu betrachten sind. Nach De lore und Le r i che sind die cöcalen Invaginationen (im Sinne von $\mathrm{B} \mathrm{l}$ a u el) die weitaus häufigsten. Ganz abgesehen von diesen Ergebnissen des kasuistischen Literaturstudiums sieht B l a u e 1 eine Stütze für seine Auffassung in den feineren anatomischen Verhältnissen, welche die Darmmuskularis im Bereiche der Bauhinschen Klappe zeigt. Nach L u s c h k a, $\mathrm{H}$ e n l e und $\mathrm{K}$ r a us besteht die Valvula Bauhini aus der Schlejmhaut und Ringmuskelschicht, während die Längsmuskelfasern des Dünndarmes, an Stärke zunehmend, auf die Wand des Dickdarmes übertreten. Nach diesen Verhältnissen wäre eine Einstülpung der Klappe in das Cöcum durch $Z$ ug der Längsmuskelfasern sehr leicht verständlich. Nun beweisen aber die schönen Untersuchungen $\mathrm{T}$ o $1 \mathrm{~d} \mathrm{t} \mathrm{s}$, daB sowohl vom Ileum wie vom Dickdarm ein gewisser Teil der Längsmuskelfasern in die Klappe übergeht, wo sie zwischen den Ringmuskelschichten bis nahe an die Klappenlippe heran verlaufen. Diese Anordnung der Muskulatur verhindert leicht ersichtlicherweise geradezu eine Invagination der Klappe; sie hat eher eine Eversion bei Zug der Längsmuskeln zur Folge. Ohne allen Deduktionen B la u e ls zu folgen, kann man jedenfalls so viel sagen, daß die Klappe gemäß der Disposition ihrer Muskelschichten nicht besonders $\mathrm{zu}$ Invaginationen prädisponiert ist.

Aus der Arbeit Blauels geht jedenfalls hervor, daB eine Anzahl sog. ileócöcaler Invaginationen aus primären Einstülpungen 
der Cöcalkuppe hervorgehen, welche die Spitze des Invaginatums bildet. Dagegen halten wir den ferneren SchluB, daß dieser Invaginationsmodus der Mehrzahl aller Fälle von sog. Ileocöcalinvaginationen zugrunde liege, für unbewiesen und zuweitgehend, wenn die theoretische Wahrscheinlichkeit auch bis zu einem gewissen Grade für die Auffassung spricht. Das wesentliche Verdienst der Arbeit liegt wohl darin, auf das relativ häufige Vorkommen der Cöcumeinstülpung und auf die Unwahrscheinlichkeit einer überwiegenden primären Invagination der Ileocöcalklappe hingewiesen zu haben. Dagegen ist vielleicht ein ebensogroßer Prozentsatz von, ,ileocöcalen“ Einscheidungen der Autoren auf primäre Invagination eines kurzen Stückes Ileum zurückzuführen. UnserefünfFällebilden wohl einen direkten Beweis für diese schon von Wil ms a usgesprochene Vermutung.

Die andere Publikation aus jüngerer Zeit, welche gegen die Lehre von der Invaginatio ileocoecalis in ihrer alten Form Stellung nimmt, stammt von Prop p ing.

Auf Grund von Experimenten am Tier, die allerdings nicht wiedergegeben werden, stellt P r o p ping zunächst eine Theorie über den Mechanismus der Entstehung von Invaginationen auf. Unter Zurückweisung der Annahme einer Invaginatio paralytica oder spasmodica sowie der Besnierschen Theorie, nach der eine mäßig kontrahierte Darmstrecke infolge der Schwere des oberhalb befindlichen Darminhaltes in die tiefer gelegene rein mechanisch hineinfällt, vertritt Propping folgende Anschauung vom Invaginationsmechanismus: Die Längsmuskulatur hat bei der Invagination keine aktive Rolle; sie macht ihre Wirkung, wie aus den schematischen Zeichnungen, die der Autor bringt, hervorgeht, vielmehr in einer Weise geltend, die zur Desinvagination führen würde. Die auch von Wilms akzeptieret Erklärung $\mathrm{N}$ oth $\mathrm{n}$ a ge $\mathrm{ls}$, die Wirkung der Längsmuskulatur betreffend, wird damit zurückgewiesen. Die Invagination entsteht nach Propping dadurch, daß durch Kontraktion der Ringmuskulatur auf eine bestimmte Strecke des Darmes hin eine Verlängerung des betreffenden Darmteiles hervorgerufen wird, wodurch eine Einstülpung dieser in die nächst anale und orale Partie zustande kommt. So entsteht eine minimale Überdachung und damit ist der Anfang der Invagination gegeben. Die weitere Einscheidung erfolgt allein durch die Wirkung der Ringmuskulatur. P r o p p in g lehnt sich dabei an eine schon von $\mathrm{Br}$ int on aufgestellte Hypothese an. Die Invaginatio iliaca, sowie 
die Invaginatio colica sind durch diese Theorie ohne weiteres zu erklären; Schweirigkeiten macht dagegen die ileocöcale Form. Hier ist die Entstehung der Einscheidung durch Herüberstülpen der Scheide über den kontrahierten Darm nicht annehmbar, weil ja die Ringmuskulatur des Dünndarmes und diejenige des Dickdarmes so ziemlich im rechten Winkel zueinander stehen. Propping operierte nun auch drei Fälle (Kinder), von denen bei zweien nicht das Ileocöcalostikum, sondern eine Partie zwischen vorderer und seitlicher Tänie des Cöcums die Spitze der Invagination bildete. Unter Hinweis auf die Arbeit von Lor enz führt deshalb Propping die Ileocöcalinvaginationen auf den gleichen Mechanismus zurück, wie die Dünndarminvagination: „eine tetanische Kontraktion des Cöcums mit der damit verbundenen Verlängerung des Organs schafft die - anale schirmförmige Überdachung, und die fortschreitende spastische Kontraktion der Ringmuskeln des überwölbenden Darmteiles führt die eigentliche Invagination herbei. Die Vorstellung von der Entstehung einer Cöcalinvagination verliert sofort ihre Schwierigkeit, wenn man das Ileum und seine Einmündung ganz außer acht läßt, was bei der rein passiven Rolle des Dünndarmes eine natürliche Berechtigung hat. Je nachdem, in welcher Höhe die erste tetanische Kontraktion ihre Grenze findet, wird bald das Ileocöcalostium, bald ein anderer Teil des Cöcums zur Spitze der Invagination.“

Wie man sieht, spricht sich Propping hinsichtlich der Ileocöcalinvaginationen nicht so extrem aus wie Bla uel. Er läßt sie bestehen, aber nur als rein zufällige Varianten einer Cöcaleinscheidung, deren Häufigkeit bei Kindern P r o p p ing damit erklärt, $d a B$ bei ihnen die Speisen etwa 24 Stunden im Cöcum verweilen, das Cöcum somit ein stets tätiges Organ darstelle, in dem deshalb auch die meisten Störungen der Darmbewegungen stattfinden können.

Durch diese beiden neueren Arbeiten hat meines Erachtens die Lehre von der ileocöcalen Invagination einen empfind lichen StoB be k o m m e n. Wenn wir auch nicht so weit gehen wie B l a u e 1 , der die ileocöcale Form nur als Ausnahme gelten lassen will und den Beginn der Invagination beinahe ausnahmslos in die Kuppe des Cöcums verlegt, so verdient doch das klinische Material, welches er beibringt, lebhafte Beachtung, und auch seinen anatomischen Exkursen kann man sich nicht ganz entziehen. Die Ausführungen Prop ping s bilden einen weiteren kasuistischen Beitrag zu den Beobachtungen Blauels und können als ex- 
perimentelle Ergänzung der von $\mathrm{B} 1$ a u e 1 vorgetragenen klinischliterarischen Deduktionen angesehen werden. Beide Arbeiten zeigen jedenfalls, da $B$ unsere bisherigen Anschauungen über das Vorkommen und die Art der Entstehung der eigentlichen Invaginatio ileocoecalis (Valvulae $\mathrm{B}$ a $\mathrm{u}$ h in i) durchaus mangelhaft begründet, zum Teil sicher falsch, jedenfalls aber revisionsbedürftig sind. Immerhin scheinen mir beide Arbeiten der Kritik zugänglich zu sein. B l a u e ls Schlußfolgerungen basieren zum Teil auf der Annahme der Nothnagelschen Regel, nach welcher der Ort, wo die primäre Invagination stattfand, auch später stets an der Spitze der Einstülpung bleibe. Diese Regel, welche jedes aktive Vordringen der invaginierten Schlinge ausschlieBt, scheint mir in ihrer abstrakten Form nicht zutreffend zu sein; die bisherigen Experimente sind jedenfalls nicht beweisend und bedürfen um so mehr der Nachprüfung, als in den Proppingschen Versuchen, so einleuchtend die Ausführungen des Verfassers namentlich im kritischen Teil der Arbeit sind, auch nur das Anfangsstadium der Invagination, die schirmförmige Überdachung, beweisend ist, während die Erklärung der weiteren Entwicklung der Einstülpung auf, allerdings geistreicher, Hypothese beruht. Das Thema bedarf noch weiterer experimenteller Abklärung. Ohne derselben vorzugreifen, können wir sagen, daß die Annahme, bei der Invaginatio ileocoecalis gehe stets die Bauhinische Klappe voran, nicht allgemeine Gültigkeit hat. Wie die Zusammenstellung $\mathrm{Bla}$ u els beweist, geht in einer Anzahl von Fällen das Cöcum voran, während unsere fünf Betrachtungen zeigen, $\mathrm{da} B$ auch primär kurz vor der Klappe entstandene Ileumeinstülpungen, die sich zunächst in ileocolische umwandelten, durch sekundäres Nachziehen des Cöcums mit Appendix zu sog. Invaginationes ileocoecales werden können. $\mathrm{D}$ ur $\mathrm{ch}$ d i es e beid e $n, b$ is h e r $z$ u wenig beachteten Formen wird das Gebiet der Invagination mit Vorantritt des Valvula Bauhini schon wesentlich eingeschränkt. Dazu kommt nun ferner, daß die sog. anatomischen disponierenden Momente, die zur Erklärung des überwiegenden Auftretens der Ileocöcalinvagination im Kindesalter herangezogen wurden, der Kritik nicht standhalten. Die anatomische Disposition der Muskulatur im Bereich der Klappe, die rechtwinklige Stellung der Ringmuskel- 
ebene des Dünndarmes zu derjenigen des Dickdarmes scheinen vielmehr der Entstehung einer Invaginatio Valvulae Bauhini direkt hinderlich zu sein. Die Annahme Proppings, daß ein fernerer Teil der ileocöcalen Einscheidungen im Bereiche des Cöcums und Colon ascendens entsteht (exklusive Cöcalkuppe, die nur passiv nachgezogen wird), scheint mir deshalb sehr plausibel und erklärt wieder eine Anzahl ,ileocöcaler" Einscheidungen, ohne da $B$ wir auf die anatomische Disposition rekurrieren müssen. So schränkt sich das Gebiet der Invaginatio ileocoecalis sensu strictiori noch wesentlicher ein. Ihr Vorkommensteht a u Ber Z weifel, bildet aber nicht in dem Maßedie Regel, wie $m a n$ bisher allgemein angenommen hat. Die Häufigkeit der ileocöcalen Einscheidungen in der Literatur erklärt sich nun wesentlich besser; wir haben gesehen, daß, ,ileocöcale“ Invaginationen aus iliacalen, cöcalen und colischen hervorgehen können, daß es also eine ganze Reihe von ,ileocöcalen" Einscheidungen gibt, bei denen keineswegs die Valvula Bauhini vorangeht. Die Subsummierung dieser erst sekundär zur ileocöcalen gewordenen Einscheidungen unter den großen Begriff der Invaginatio ileocoecalis beruht auf mangelhaften Unterscheidungen seitens der Autoren; deshalb geht es auch nicht an, aus der ganz allgemein gehaltenen Angabe ,ileocöcal" darauf zu schließen, daß eine echte primäre Ileocöcalis vorgelegen habe, bei der Einstülpung somit das Ostium vorangegangen sei. Die Tatsache, daß die Bezeichnung Invaginatio ileocoecalis einen $\mathrm{S}$ a $\mathrm{mmel-}$ $\mathrm{b}$ e $\mathrm{g} \mathrm{r}$ if $\mathrm{f}$ darstellt, wie wir ausgeführt haben, erklärt jedenfalls schon an sich bis zu einem gewissen Grade die Häufigkeit dieser Einscheidungsform in den statistischen Zusammenstellungen. So bleibt nur noch zu erklären, warum die Invaginationen des untersten Dünndarmes, sowie diejenigen des Cöcums und Colon ascendens in allen Lebensaltern, besonders aber bei Kindern und Jugendlichen prozentual so stark überwiegen. Wir haben schon gesehen, daß es mit den sog. anatomischen Dispositionen nicht besonders gut bestellt ist für die Erklärung dieser Tatsache, abgesehen davon, da $B$ eine Invagination des Cöcums nach oben in das Colon ascendens nur möglich ist bei Patienten, die ein freies Cöcum oder ein langes Mesenterium des Cöcums und untersten 
Teiles des Colon ascendens haben; in diese letztere Gruppe gehören die Fälle mit Mesenterium commune. Nun haben L e r i c h e und $\mathrm{C}$ ava $1110 \mathrm{n}$ in einer anatomisch-statistischen Bearbeitung dieser Frage darauf hingewiesen, da $B$ bei Föten und Neugeborenen in 45 Proz. der Fälle ein freies Cöcum, in 3,5 Proz. ein langes Mesenterium vorhanden ist, daß also 48,5 Proz. die anatomischen Vorbedingungen für das Zustandekommen einer sog. ileocöcalen Einscheidung aufweisen, während bei Erwachsenen freies Cöcum und langes Mesenterium nur in je 8,5 Proz., also zusammen in I7 Proz, der Fälle vorkommen. Dieses Untersuchungsergebnis scheint uns sehr geeignet, das Úberwiegen der ,ileocöcalen“ Einscheidungsformen bei Kindern zu erklären. Warum aber die Invaginationen des untersten Ileum, des Cöcum und des Colon ascendens überhaupt häufiger vorkommen als die Einscheidungen des übrigen Dünn- und Dickdarmes, hat dadurch noch keine Abklärung erfahren. Hier sind offenbar noch andere Momente maßgebend. Ich möchte in dieser Hinsicht auf folgende Punkte hinweisen: Im Bereiche des Ostium ileocoecale haben wir einen Sphinkter und eine klappenähnliche Vorrichtung; beide hindern den Rücktritt von Dickdarminhalt in den Dünndarm, der Sphinkter allein den zu frühen oder zu reichlichen Übertritt von Dünndarminhalt in den Dickdarm. Reizung des Nervus splanchnicus, welche die Dünndarmbewegung hemmt, ruft zugleich Kontraktion des Sphinkter ileocoecalis hervor. Wahrscheinlich sammelt sich im untersten Teil des Ileums stets eine gewisse Menge Darminhalt an, bevor sich der Sphinkter öffnet, wobei dann stets das unterste Ileum in lebhafte Peristaltik gerät. Der kontrahierte ileocöcale Sphinkter bildet für die normale Peristaltik ein Hindernis, und während nun jede Widerstandsvermehrung eine Steigerung der Peristaltik hervorzurufen geeignet ist, haben wir in der gleichzeitig mit der Sphinkterkontraktion eintretenden Hemmung des Ileums einen physiologischen komplizierten Reflexmechanismus vor uns, der diese Steigerung der Dünndarmperistaltik normalerweise verhindert. Wir sehen aber, daß dieser komplizierte Mechanismus eine $\mathrm{ph}$ y s i olog is che Disposition für die Entstehung von Invagi$\mathrm{n}$ ationen schaffen muß, insofern, als das periodische Auftreten stärkerer Peristaltik in diesem Darmgebiet bei der Mög- 
lichkeit einer Störung im normalen Ablauf des komplizierten Reflexmechanismus (Nichteintreten oder zu spätes Eintreten der Sphinkterhemmung) wohl öfters Bedingungen schafft, wie sie zur Entstehung einer Invagination notwendig sind, als das im oralen Dünndarmbezirk der Fall ist, wo dem normalen und ungehemmten Ablauf der peristaltischen Welle keine Hindernisse entgegentreten. $\mathrm{Ob}$ man nun auf dem Boden der $\mathrm{N}$ ot h $\mathrm{n}$ a g e $\mathrm{l}$ schen, von Wilms bestätigten, experimentell begründeten Theorie steht, nach der durch die Tätigkeit der Längsmuskulatur der unterhalb gelegene Darm über eine orale krontrahierte Darmpartie bogenförmig hinübergezogen wird, oder mit Propping die primäre Einstülpung (schirmförmige Überdachung) sowohl, als auch das sekundäre Größerwerden einer Invagination, nur als eine Leistung der Ringmuskulatur ansieht, i n b e i d e $\mathrm{n} F$ ä 11 e $\mathrm{n}$ ist eine gesteigerte, unregelmäBige, nicht dem gewöhnlichen Ablauf von Erregung und Hemmung entsprechende Peristaltik unbedingte Voraussetzung, und in diesem Sinne, nicht etwa in Anlehnung an Schriddes spekulative Hypothese, möchten wir unsere Darstellung aufgefaßt wissen. Die speziellen physiologischen Verhältnisse der ileocöcalen Peristaltik sollen nur die Basis schaffen, auf der sich der durch das Experiment nachgewiesene Invaginationsmechanismus, der doch eine Ursache haben muß, abspielen kann. Für das Cöcum und Colon ascendens sind solche physiologische Prädispositionen ebenfalls unschwer nachzuweisen, Bekanntlich ist am Cöcum, Colon ascendens und promaximalem Colon transversum eine Antiperistaltik nachgewiesen, die einsetzt, sobald sich der Dickdarm vom Dünndarm her mit Inhalt füllt und die offenbar der weiteren Durchknetung und Durchmischung, sowie der Eindickung des Dickdarminhaltes dient. Es ist nun leicht ersichtlich, daß durch Zusammentreffen von peristaltischer und antiperistaltischer Welle Verhältnisse entstehen können, die das Zustandekommen einer Einstülpung oder Überdachung nach Nothnagel-Wilmsschem oder Proppingschem Mechanismus ermöglichen oder direkt hervorrufen. -Das ganze Darmsegment vom untersten Ileum bis zum. Colon transversum birgt somit in seinem normalen physiologischen 
Mechanismus Invaginationsprädispositionen, welche die relative Häufigkeit der Einscheidungen in diesem Darmabschnitt unserem Verständnis näher rücken. Ich möchte diese Dispositionen, die bisher nur andeutungsweise und nur für das Cöcum ber ücksichtigt sind, physiologische nennen, und halte ihre Rolle für ungleich wichtiger, als diejenige der anatomischen Dispositionen, um so mehr, als die physiologischen Dispositionen direkt veranlassende, auslösende Momente jnvolvieren, während die anatomischen nur die Möglichkeit $\mathrm{zu}$ pathologischer Darmverlagerung schaffen (langes Mesenterium).

Auf die Kritik der Invaginationsexperimente können wir hier nicht eingehen; wir behalten sie einer experimentellen Studie vor, welche namentlich auch die Frage beantworten soll, ob die Vergrößerung der initialen Úberdachung oder Einscheidung nur auf Kosten des äuBeren analen Darmes stattfindet oder zum Teil auch der innere, orale Darm sich aktiv weiter einstülpt. Bis heute gilt allgemein die von $\mathrm{N}$ ot h $\mathrm{n}$ a g e 1 aufgestellte Regel, daß dasjenige Darmsegment, welches den ursprünglichen Ausgangspunkt der Invagination gebildet hat, auch an der Spitze der Einscheidung bleibt. Auf diese Annahme stützt sich auch $\mathrm{W}$ i $1 \mathrm{~ms}$, wenn er vorschlägt, die Invaginationen entsprechend dem Teil zu benennen, der bei der b e g i $\mathrm{n} n$ e $\mathrm{n} d \mathrm{e} n$ Invagination die Spitze bildet. Nun beobachten wir aber die Entstehung der Einscheidung nicht direkt, und wir können logischerweise aus der fertigen Einscheidung auch nicht mit absoluter Sicherheit sagen, wo der Ort der primären Invagination lag. Wie wir gesehen haben, herrschen nun besonders hinsichtlich des Sammelbegriffes der Invaginatio ileocoecalis ziemlich unklare und wenig übereinstimmende Anschauungen, und wir haben in den Arbeiten von $\mathrm{B}$ l a u e 1 $\mathrm{H}$ a n sen, Propping und in diesem kasuistischen Beitrag drei klare Einscheidungsformen kennen gelernt, die von der eigentlichen Ileocöcalis getrennt werden müssen. Wenn wir dieses Kapitel abklären wollen und namentlich auch entscheiden, ob die 
von B la uel und Propping negierte primäre Einstülpung der Valvula Bauhini (eigentliche Ileocöcalis s. s.) deren Vorkommen wesentlich einzuschränken wir gute Gründe haben, überhaupt öfters vorkommt, dürfen wir der Nomenklatur der Invaginationen keine aprioristischen Annahmen zugrunde legen. Wir dürfen deshalb eine Invagination nicht bezeichnen nach dem Orte der primären Einstülpung, auf den wir ja nur mehr oder weniger gesicherte Rückschlüsse machen können, sondern müssen eine Bezeichnung wählen, aus der man die an der Invagination beteiligten Darmabschnitte, und zwar sowohl die Komponenten des Invaginatums wie auch diejenigen der Scheide ohne weiteres herauszulesen in der Lage ist. Dann hört die Diskussion über den vorangehenden Darmteil von selbst auf. In Resektionspräparaten macht die Feststellung von Hals und Kopf keine Schwierigkeiten, und wir sehen beispielsweise auch, ob eine sog. Invaginatio ileocolica aus einem Schleimhautprolaps nach Leichtenstern oder aus einer primären Iliaca (nach W i $1 \mathrm{~m} \mathrm{~s}$; und eigenem Falle) hervorgegangen ist (Wandkomponenten des inneren und mittleren Zylinders an der Spitze!). Bei Desinvaginationen haben wir an den sekundären Darmveränderungen genügende Anhaltspunkte für die Feststellung der Spitze; gewöhnlich kommt diese Umschlagsstelle ja auch zuletzt zum Vorschein und setzt der Entwicklung die größten Schwierigkeiten entgegen. Wir sind deshalb bei operativ behandelten Fällen, und diese allein sind maßgebend, stets genau darüber orientiert, welcher Darmteil Hals, Kopf, Scheide und Intussuszeptum der Invagination bildet und es handelt sich nur darum, die Bezeichnung der Einstülpung so zu wählen, daß sie die genaue Topographie der Invagination ohne weiteres abzulesen gestattet. Um dieses Postulat zu erfüllen, bringen wir folgendes in Vorschlag: Invaginationen, bei denen es sich um Einstülpung eines Darmteiles ingleichnamigen Darm handelt, werden, wie bisher, mit einem einteiligen Wort be $z$ eichnet, also eine Einstülpung von Ileum in Ileum als Invaginatio iliaca, eine Einstülpung von Colon in Colon als Invaginatio colica. F ï $r$ alle anderen Intussuszeptionen, bei welchen das Intussuszeptum oder die Scheide oder bejde zusammen von mehr als einem Darm- 
teil gebildet werden, wählen wir eine zweiteilige Bezeichnung, deren erster Teil den oder die Komponenten des Invaginatum, deren zweiter Teil die Komponenten der Scheide nennt. Derjenige Darmteil, welcher den Kopf der Invagination bildet (nach Noth$\mathrm{n}$ a g e $\mathrm{l}$ die Stelle der primären Einstülpung), wi $\mathrm{rd}$ a u ch in der Bezeichnung an die Spitze gestellt. Für die Invaginatio ileocoecalis gestalten sich die Verhältnisse dann folgendermaßen: Die eigentliche Invaginatio ileocoecalis mit Vorantritt des Ostiums behält die von $\mathrm{W}$ il ms vorgeschlagene Bezeichnung Invaginatio ileocoecalis - Valvulae Bauhini. Eine Einstülpung des Ileums in das Colon mit Nachziehen des Cöcums nennen wir Invaginatio ileocoeco-colica, den von Bla u el u. a. beschriebenen Typus mit Vorangehen der Cöcalkuppe und Nachziehen des Ileums - wobei also das Ostium ileocoecale seitlich an der Kuppe sitzt - bezeichnen wir als Invaginatio coecoileo-colica. Geht z. B. die primär invaginierte Ileumpartie durch das Colon bis in das S. Romanum unter Nachziehen des Cöcums, so würde die genaue Topographie dieser Einstülpung durch die Bezeichnung Invaginatio ileocoecocolosigmoidea wiedergegeben. Doppelte Einscheidungen im gleichen Niveau, also fünf- und siebenzylindrische Einschiebungen, werden durch den Zusatz duplex resp. triplex genauer charakterisiert. Nach dieser neuen Art der Bezeichnung können wir somit auch komplizierte Invaginationen sehr genau wiedergeben, wie an dem Beispiel unseres Falles $5 \mathrm{zu}$ ersehen ist: Es handelte sich um Einstülpung des Ileum durch das Colon ascendens und transversum bis zum Colon descendens unter Nachziehen des Cöcums, dazu um Invagination des Sigmoideum in das Rektum, also um eine Invaginatio ileocoeco-colica und sigmoidearectalis, duplex (fünfzylindrisch). Umstülpung des Appendix sowie aszendisender Verlauf der Invagination müssen natürlich besonders angeführt werden.

Wir sind uns wohl bewußt, init diesem Vorschlag keine Vereinfachung der Nomenklatur zu bringen, dagegen glauben wir in der Art der Bezeichnung der einzelnen Invaginationen größere Klarheit zu schaffen; Unklarheiten, wie sie z. B. durch die Sammel- 
bezeichnung Invaginatio ileocoecalis bedingt werden, kommen vollständig in Wegfall. Ferner trägt die neue Nomenklatur den bisher zu wenig beachteten Formen, bei denen die Cöcalkuppe, ein Teil des übrigen Cöcums oder eine kurz vor der Bauhinschen Klappe entstandene Iliacaleinscheidung an der Spitze einer sog. Invaginatio ileocoecalis marschiert, in gebührender Weise Rechnung.

Demnach wären folgende Hauptformen von Darmeinscheidungen zu unterscheiden:

I. Invaginationes entericae (jejunalis, iliaca, ev. Kombination zwischen jejunalis und iliaca).

2. I. ileo-colica (aus einer I. iliaca hervorgegangen).

3. I. ileocoeco-colica (sekundäres Nachziehen des Cöcums).

4. I. coecalis (Cöcum in Cöcum).

5. I. coecoileo-colica (sekundäres Nachziehen des Ileums).

6. I. ileocoecalis s. strict. - Valvulae Bauhini1).

7. I. colica.

Unter den Coloninvaginationen können nach unserer Nomenklatur noch fernerhin unterschieden werden: Invaginatio colosigmoidea, Invaginatio sigmoideo-rectalis, Invaginatio colosigmoideorectalis und ev. der eigentliche Prolapsus recti als Invaginatio rectalis.

Diejenigen Formen, bei denen eine Dünndarm- oder Cöcalinvagination nicht nur bis in das Colon, sondern bis in den Bereich des Sigmoideum und Rektum vorgedrungen ist, nicht speziell erwähnt. Ihre Bezeichnung ergibt sich aus den vorstehenden Erklärungen. Auch die theoretisch mögliche Invaginatio jejunoileocolica haben wir nicht besonders aufgeführt, da von den reinen Dünndarm-Coloneinscheidungen ohne Nachziehen des Cöcum nur die Ileo-colica praktisch eine Rolle spielt. Ferner hielten wir es nicht für notwendig, die beiden Formen der primären Cöcalinvaginationen, die von $\mathrm{B}$ l a $\mathrm{u}$ e $\mathrm{l}$ beschriebene mit Vorangehen der Kuppe und die von Propping beobachtete, bei der ein höher, d. h. näher der Ileocöcalklappe gelegener Teil des Cöcums die Spitze bildete, auch noch speziell auseinander zu halten. Man wird beide Typen als Invaginationes coeco-colicae, ev. coecoileo-colicae be-

I) Fälle mit Schleimhautprolaps nach Leichtenstern sollten speziell beschrieben werden, falls solche uberhaupt beobachtet werden, gehören aber zu Nr. 6 . 
zeichnen und kann eventuell die Blauelsche Form im gegebenen Falle noch näher charakterisieren als ,Invaginatio coecoileo-colica (apicis)". Das Wesentliche scheint uns die Angabe zu sein, daß das $\mathrm{C}$ ö c u m vorangeht, während es auf das Niveau, in welchem die primäre Cöcalinvagination erfolgte, bei dem meist recht kurzen Blinddarm weniger ankommen dürfte. Das aufgestellte Schema wird allen wesentlichen praktischen Bedürfnissen genügen. Ganz seltene Formen können nach den der neuen Nomenklatur zugrunde gelegten allgemeinen Regeln, wie wir sie aufgestellt haben, ohne Schwierigkeiten prägnant bezeichnet werden. - Zusammenfassend sei auf die wesentlichen Ergebnisse dieser Arbeit hingewiesen:

In Bestätigung bekannter Erfahrungen zeigt unser Material, daß man die Diagnose Darminvagination nicht vom Nachweis eines Invaginationstumors abhängig machen darf; wo das Dancesche Symptom vorhanden ist, hat man Grund, eine Verschiebung des Tumors nach oben unter den Rippenbogen anzunehmen, während zunehmender Meteorismus auch einen in den Anfangsstadien der Erkrankung nachweisbaren Tumor der Palpation entziehen kann, auch in Narkose.

Die Prognose der Resektion ist eine schlechte, weil der Eingriff von Kindern nicht gut vertragen wird, und weil es die weiter vorgeschrittenen Fälle sind, welche dieser Operation unterworfen werden müssen.

Die Lösung der Invagination hat sehr oft eine Einschwemmung von Bakterien und Toxinen aus der veränderten Darmwand in die Blut- und Lymphwege zur Folge, ein Vorgang, der zu hohen postoperativen Temperatursteigerungen führt und Exitus letalis bedingen kann. Forcierte Desinvaginationsversuche sind deshalb zu unterlassen.

Die Lehre von der Invaginatio ,ileocoecalis“ bedarf der Revision; durch die von Blauel und Propping beigebrachten Fälle von primärer Cöcalinvagination sowie durch unsere Beobachtungen, welche die Entstehung ileocöcaler Einscheidungen aus kurzen Iliacalinvaginationen demonstrieren, wird das Gebiet der eigentlichen Invaginatio ileocoecalis mit Vorangehen der Klappe wesentlich eingeschränkt. 
Die viel diskutierten anatomischen Dispositionen, welche zur Erklärung der Häufigkeit ileocöcaler Einscheidungen konstruiert wurden; halten nur zum kleinsten Teil der Kritik stand; ungleich wichtiger sind die bisher zu wenig berücksichtigten physiologischen Prädispositionen, welche im normalen Mechanismus der Peristaltik des ganzen Darmsegments vom untersten Ileum bis zum Colon transversum gegeben sind und direkt veranlassende Momente darstellen, wo die anatomischen Dispositionen nur die Möglichkeit zu pathologischer Darmverlagerung schaffen.

Unsere bisherige Einteilung und Nomenklatur der Invaginationen trägt den einzelnen Typen der ileocöcalen Einscheidung nicht genügende Rechnung. Im Interesse einer Abklärung der Frage ist deshalb eine Bezeichnungsart zu wählen, welche gestattet, die Invaginationen genau zu charakterisieren und die einzelnen Komponenten in ihren gegenseitigen topischen Beziehungen aus der Bezeichnung ohne weiteres herauszulesen. Für diese neue Nomenklatur bringen wir bestimmte Vorschläge und legen ihr die fertige Intussuszeption zugrunde, da eine Bezeichnung nach dem Orte der primären Einstülpung ungenau sein muß und gewisse Präjudize schafft. Falls in den künftigen kasuistischen Mitteilungen diese genauere Charakterisierung der Invaginationen Anwendung finden sollte, wird der Anteil der einzelnen, zum Teil erst in neuester Zeit berücksichtigten Typen an der großen Gruppe der Invaginatio ileocoecalis sich bestimmen lassen und wir werden namentlich auch erfahren, ob ein Schleimhautprolaps nach $\mathrm{L}$ e i c $\mathrm{h}$ te nstern oder eine primäre Klappeneinstülpung resp. Fälle, bei denen die Valvula Bauhini an der Spitze der Invagination sich befindet, überhaupt und in nennenswertester Weise vorkommen. Úber diese Frage vermögen nur unsere Beobachtungen intra operationem Auskunft zu geben; nach dem bisher üblichen Bezeichnungsmodus erhalten wir jedoch über wesentliche Punkte keine brauchbare Auskunft, weshalb der Sammelbegriff der Invaginatio ileocoecalis aufzugeben und durch eine genauere Nomenklatur zu ersetzen ist, deren Anwendung auch auf die übrigen Einscheidungen sich im Interesse der Klarheit von selbst empfiehlt. 


\section{Literaturverzeichnis.}

(Vollständiges Literaturverzeichnis bei $\mathrm{W}$ i $1 \mathrm{~ms}$, ,Der Ileus“.)

I. A l a p y, H., Der Darmverschluß der Kinder. Archiv f. klin. Chirurgie rgio, Bd. 9I.

2. B l a u e 1, Zur Mechanik der Invaginatio ileocoecalis. Beiträge zur klin. Chirurgie, Bd. 68, Heft I.

3. B r a u n, Invaginatio ileocoecalis. Berichte der med. Gesellschaft zu Leipzig, Juli 1897 .

4. Dersel be, Darminvagination. 75. Naturforscherversammlung. Münchn. med. Wochenschr. 1903, Nr. 39.

5. C h e $\mathrm{n}$ e y, F., Intussusception in an infant etc. New York med. News I 895.

6. $\mathrm{C} 1 \mathrm{u} \mathrm{b} \mathrm{b} \mathrm{e,} \mathrm{Notes} \mathrm{on} \mathrm{fifteen} \mathrm{cases} \mathrm{of} \mathrm{intussusception.} \mathrm{British} \mathrm{med.} \mathrm{Journ.} \mathrm{I897.}$

7. Derselbe, Ch., (Sidney). British med. Journ. I907.

8. D a n ce, Mém. sur les invaginations morbides des intestins, Répertoire génerale d'anat. et de phys. etc. Paris 1826 (zitiert nach Wilms).

9. Der se $1 \mathrm{be}$, Observat. sur une invagin. intest. (zitiert nach Wilms).

Io. Delore et $\mathrm{L}$ e rich e, Sur la pathogénie et le traitement de l'invagination intestinale. Revue de Chirurgie I 908.

I I. Deville chabrolle, Des invaginations appendico-coecales. Thèse de Bordeaux 1908 .

I2. v. Eis els berg, Zur radikalen Behandlung der Darminvagination; 74. Naturforscherversammlung 1902. Zentralbl. f. Chirurgie 1902, Nr. 48.

13. Derselbe, Langenbecks Archiv I903, Heft I und 2. Festband für v. Es march.

14. Eve, T., British med. Journ. Igor, Vol. r.

15. F is c h I, Erkrankungen des Magens und Darmes im frühesten Kindesalter. Handbuch der Kinderheilkunde von Pfaundler und Schloßmann.

16. F a g g e, Intussusceptio in Infants, Practitioner 1906.

I7. Gibson, L., Mortality and treatment of acute intussusception. Med. Record $\mathrm{i} 897$.

18. Ha nse n, P., Die Behandlung der akuten Darminvagination im Kindesalter. Mitteil. a. d. Grenzgebieten der Medizin u. Chirurgie, Bd. 18, Heft I.

19. Hirschsprung, Beobachtungen über Darminvagination bei Kindern; Jahrbuch f. Kinderheilkunde I895, Bd. 39, Heft 4.

20. D e r s e l b e, IO Fälle von Invagination bei Kindern. Mitteil. a. d. Grenzgeb. der Medizin u. Chirurgie, Bd. I4.

21. Karewski, Die chirurgischen Erkrankungen des Kindesalters.

22. Kle m m, Über die akute Darminvagination im Kindesalter. Deutsche Zeitschr. f. Chirurgie, Bd. 44.

23. Kra us, O., Zur Anatomie der Ileocöcalklappe. Archiv f. klin. Chirurgie, Bd. 44 .

24. $\mathrm{Kirm}$ is on, De l'invagination intestinale chez les enfants etc. Acad. de méd. Séance du 25 Juin 1907.

25. Leichtenstern, Über Darminvagination. Prager Vierteljahrsschrift I 873 und 1874 , Bd. II 8 , II9 und I 21 . 
26. Leriche et Cavaillon, Semaine médicale I907, Nr. 8.

27. L or en z, H., Ein Beitrag zur Lehre von der Invagination. Deutsche Zeitschrift f. Chirurgie, Bd. 77 .

28. L u s h ka, Die organische Muskulatur innerhalb verschiedener Falten des menschlichen Bauchfells. Archiv f. Anatom., Physiologie etc. I862.

29. Noth na ge 1, Die Erkrankungen des Darmes und des Peritoneums. Wien 1903 .

30. P ower, D'A r c y, On the pathology and surgery of intussusception. The Lancet 1897 , Heft I.

3I. Derselbe, Intussusception. The Lancet 1899 , Heft $x$.

32. Prop ping, Über den Mechanismus der Darminvagination. Mitteil. a.d. Grenzgeb. der Medizin u. Chirurgie, Bd. 2I, Heft 3.

33. Rydygier, Zur Behandlung der Darminvaginationen. Verhandl. des Chirurgenkongresses 1895 .

34. Derselbe, Zur Behandlung der Darminvagination. Deutsche Zeitschr. f. Chirurgie, Bd. 42 .

35. Solieri, Deutsche Zeitschr. f. Chirurgie 1910, Bd. IO7.

36. S chla $\mathrm{n}$ ge, Der Ileus; im Handbuch der prakt. Chirurgie von Bergmann und Bruns 1907 .

37. Spitzy, Invagination und Darmverschluß; im Handbuch der Kinderkrankheiten von Pfaundler und Schloßmann, I9IO, Bd. 5 .

38. Schridde, Eine nach Ausstoßung des Intussusceptums geheilte Invagination des Ileum. Münchn. med. Wochenschr. 1903, Nr. 30.

39. Toldt, Die Formbildung des menschlichen Blinddarms und die Valvula coli. Sitzungsberichte der kaiserl. Akademie der Wissenschaften, Wien 1894, Bd. 103.

40. $\mathrm{Tr}$ eves, Intestinal Obstruction. London I899.

4r. Walterman $n$, Die Laparotomie bei Darminvagination im Kindesalter Diss., Kiel 1902.

42. W e i $\beta$, Intussusceptio intestini. Sammelreferat. Mitteil. a. den Grenzgeb. der Medizin u. Chirurgie Igoo, Bd. 2, Nr. 22.

43. Wil ms, Der Ileus. Dentsche Chirurgie 1906, Lief. 46. 